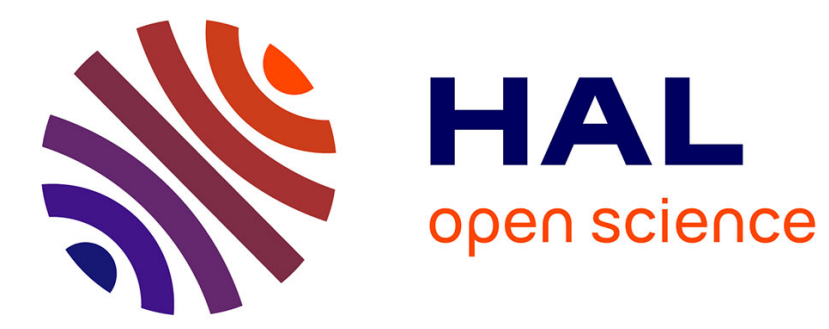

\title{
Deux fours de potier hallstattiens à Florange (Moselle)
}

Pierre Pétrequin, Jean-François Piningre, Dominique Vuaillat

\section{To cite this version:}

Pierre Pétrequin, Jean-François Piningre, Dominique Vuaillat. Deux fours de potier hallstattiens à Florange (Moselle). Gallia - Fouilles et monuments archéologiques en France métropolitaine, 1973, 31 (2), pp.267-291. 10.3406/galia.1973.2632 . hal-01926350

\section{HAL Id: hal-01926350 https://hal.science/hal-01926350}

Submitted on 4 Feb 2020

HAL is a multi-disciplinary open access archive for the deposit and dissemination of scientific research documents, whether they are published or not. The documents may come from teaching and research institutions in France or abroad, or from public or private research centers.
L'archive ouverte pluridisciplinaire HAL, est destinée au dépôt et à la diffusion de documents scientifiques de niveau recherche, publiés ou non, émanant des établissements d'enseignement et de recherche français ou étrangers, des laboratoires publics ou privés.

\section{(1) (1) $\$$}

Distributed under a Creative Commons Attribution - NonCommercial - NoDerivatives| 4.0 


\title{
DEUX FOURS DE POTIER HALLSTATTIENS A FLORANGE (Moselle)
}

\author{
par Pierre PETREQUIN, Jean-François PININGRE et Dominique VUAILLAT
}

Le site de Sainte-Agalhe sétend sur une terrasse de la vallée de la Ioselle, voie naturelle de passige qui sépare, au niveau de Florange, les cotes de Moselle du plateau lorrain, traverse plus au nord, le massif schisteux rhénan (Hunsück-Eifel) et se jette dans te Khin au niveau de Coblence. La Moselle permet un contact méridien entre la vallée du Rhin moyen et le seuil de Lorraine. La fouille ${ }^{1}$, coordonnées géographiques : $875,20 \times$ $186,60 \times 17.1 \mathrm{~m}$, Lekange 1-2, 1/20 000, I(i.) s'implante sur une haute terrasse qui domine de 30 à $35 \mathrm{~m}$ le lit actuel de la Moselle et šincline en pente légère vers le nord-ouest (fig. 1). Cette terrisse est surplombée par les côtes de Moselle et découpée au nord par la Fentch, à l'est par la Moselle et au sud par le Kirisbach (fig. 2).

La carte géologique au 1/50000 - Thionville Waldisse -- mentionne deux terrasses fluviatiles du Quaternaire récent : une haute terrasse $(+30,+35 \mathrm{~m})$ et une basse terrasse $(+15),+20 \mathrm{~m})$. Toutes deux sont couvertes de limons et de lehm. L'examen granulométrique des échantillons prélevés montre des limons sableux de teinte jaunâtre, produits de l'altération du soubassement (Lias et Keuper). I)ans les limons sous-jacents aux niveaux archéologiques, nous avons recueilli deux échantillons, a jo) et 135 $\mathrm{cm}$ de profondeur. L'examen morphoscopique des quartz permet de dresser le tableau suivant :

\begin{tabular}{|c|c|c|}
\hline profondeur de l'échantillon.......................... & $50 \mathrm{~cm}$ & $135 \mathrm{~cm}$ \\
\hline 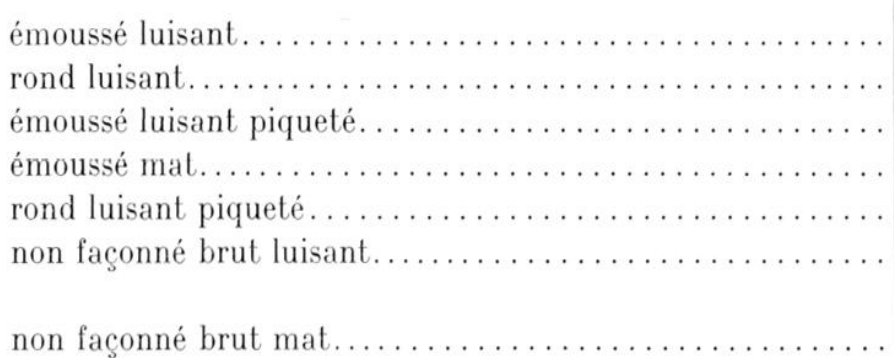 & 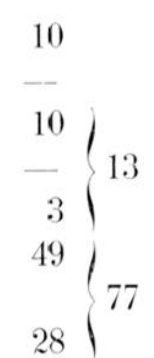 & 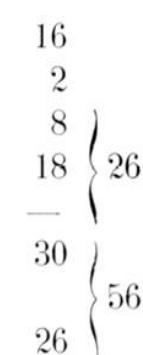 \\
\hline pourcentages... & $100 \%$ & $100 \%$ \\
\hline
\end{tabular}

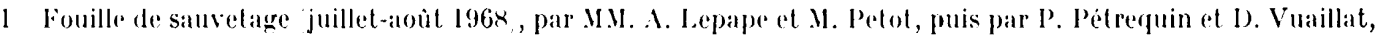
aides de .llle Baroth, P. Helias, J.-P. Lepape et J.-F. Piningre octobre 196x. 


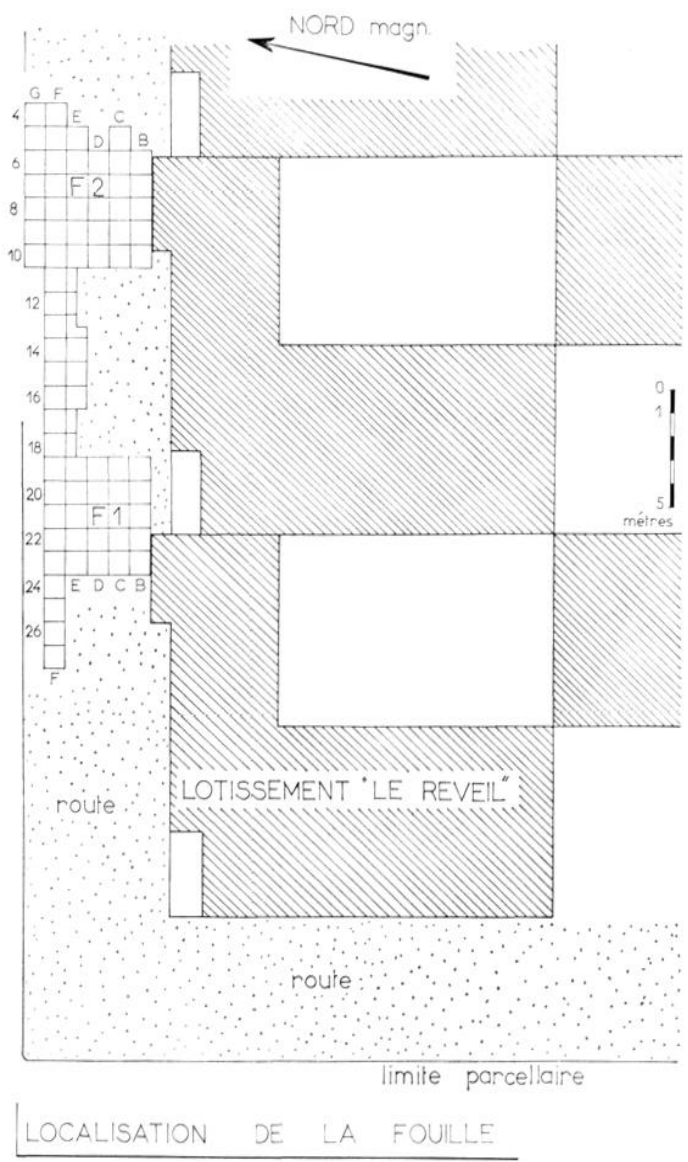

l Situation de la fouille par rapport au lotissement.

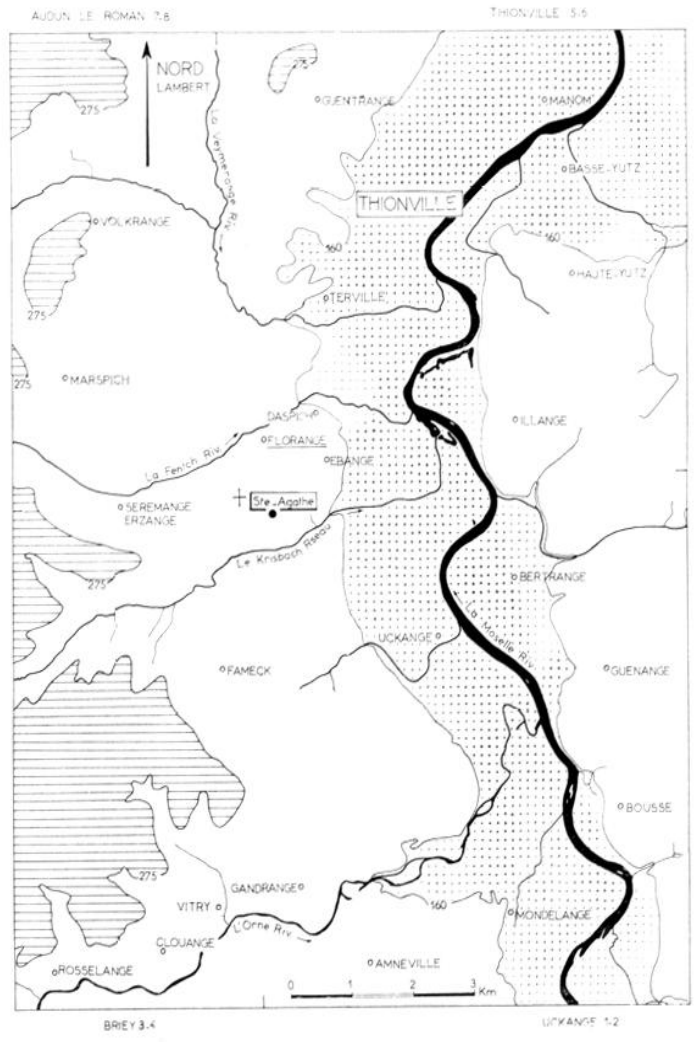

2 Lal terrasse de Florange dans son contexte orohydrographique, d'apres les cartes IGN 1/20000. En pointillés, le lit majeur de la Moselle; en blanc, les terrasse's anciennes; en lignes paralliles, les reliefs supérieurs à $275 \mathrm{~m}$ icotes de Moselle;.

La part des éléments éoliens est importante, surtout dans la couche inférieure. Il s'agit, selon toute vraisemblance, de limons d'origine fluviatile plus ou moins repris par le vent. Les éléments locaux (quartz bruts) sont nombreux et témoignent d'une mise en place par lessivage, colluvionnement et solifluction probable, au moins dans les parties superficielles des couches. Ces limons ne contiennent ni galet ni plaquette calcaire. Quoique souvent imperméables, les sols supérieurs ont été fortement lessivés à la suite de l'action des fouisseurs et de la végétation.

Le dégagement de deux stratigraphies longitudinales (D5 à 27 du carroyage) a mis en évidence deux fosses à remplissage archéologique et un sol extéricur contemporain de ces fosses. Pour mieux replacer ces structures dans leur contexte, nous avons décapé le sol extérieur sur $70 \mathrm{~m}^{2}$ (fig. 3 et 4 ).

\section{LE SOL DE L'HABITAT.}

Lin coupe (fig. 3 et 4), nous distinguons de bas en haut : le sol naturel, composí de limons argileux jaunes à nodules ferrugineux parfois concentrés en granules;

un niveau archéologique de 4 a $10 \mathrm{~cm}$ d'épaisseur. Ce sol est défini par une concentration de galets, 


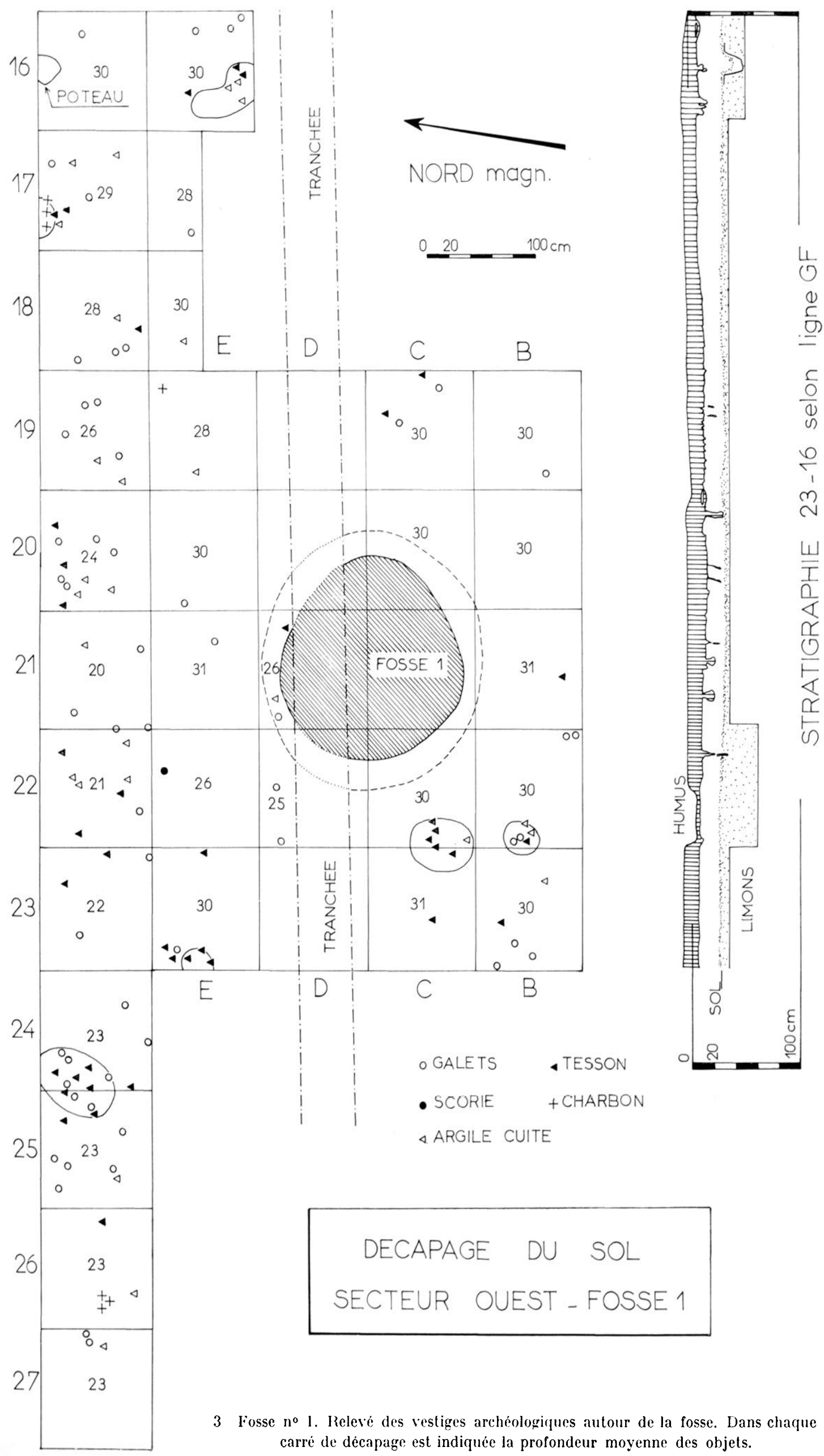




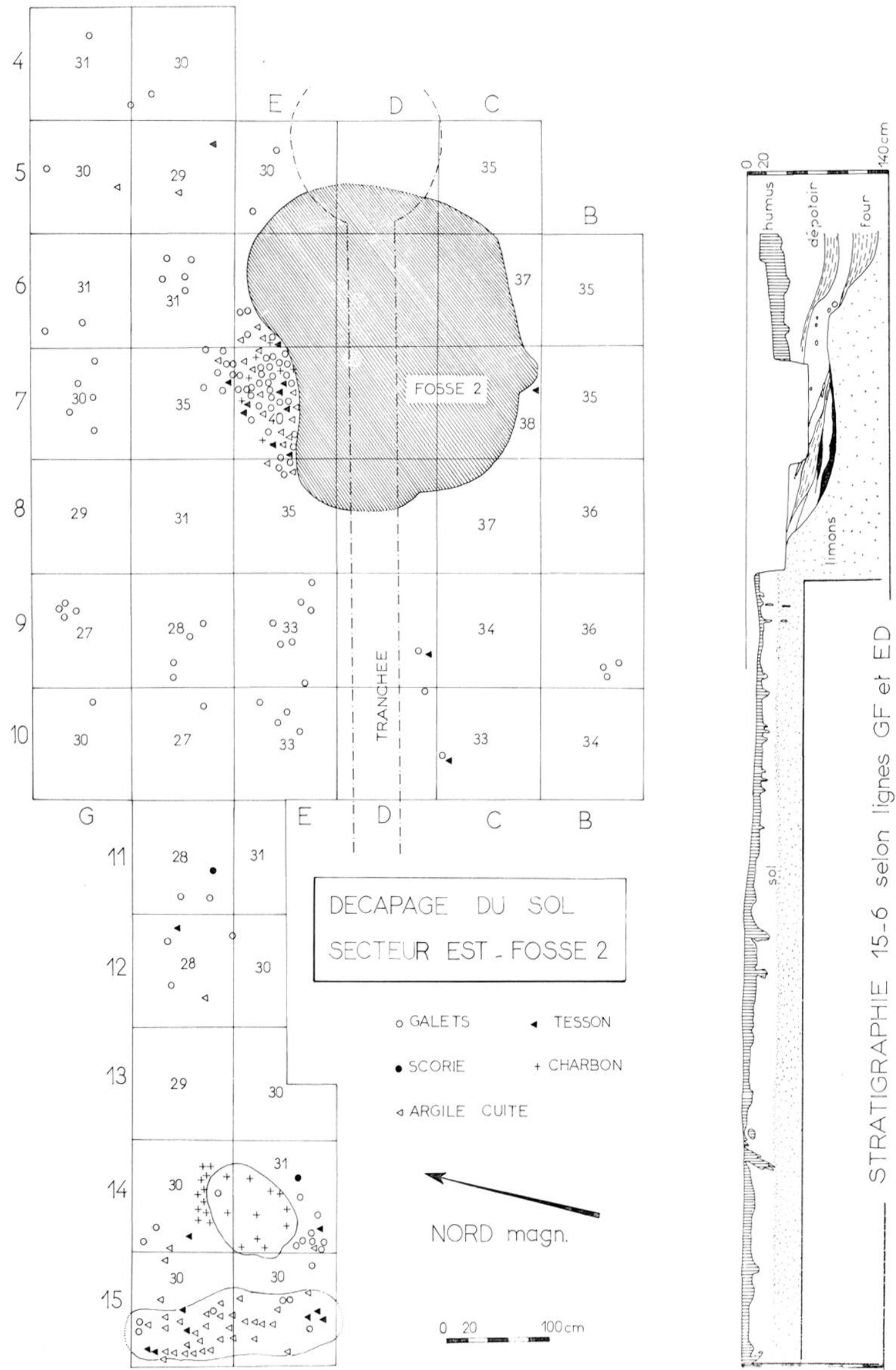

4 Fosse no 2 . Relevé des vestiges archéologiques autour de la fosse. Dans chaque carré de décapage est indiquée la profondeur movenne des objets. 


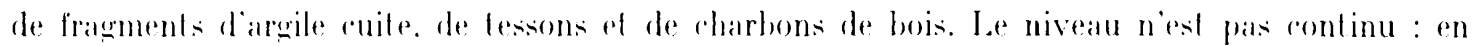
plusieurs points. il y a superposition de sols lenticulaires. In trou de poleatu rarre F lois. visible en strat igraphie. appartient lien a ce sol dhabilal. Guelques remaniements de delail terriers de

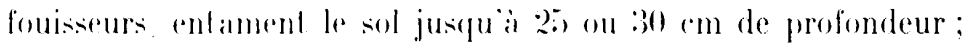

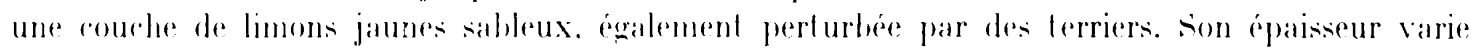
de 10 a 20 (cm. Quelques objels modernes se rencontrent dans re niveau. dans laxe des lerriers de follisiseurs:

dans la partie superticielle du gisement. un niveau humique de limons gris sableux. Il est parsemé

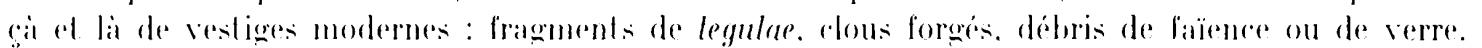

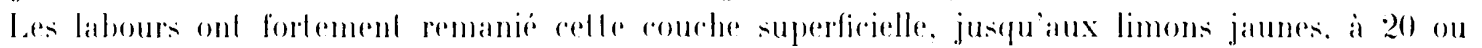
30) (om de profonderur.

L'étude morphoseopique des quartz confirme nos observations stratigraphiques :

\begin{tabular}{|c|c|c|c|}
\hline profondeur & $\begin{array}{l}\text { humus gris } \\
\text { superficiel }\end{array}$ & $\begin{array}{l}\text { limon jaune } \\
\text { intermédiaire }\end{array}$ & $\begin{array}{c}\text { sol } \\
\text { archéologique }\end{array}$ \\
\hline 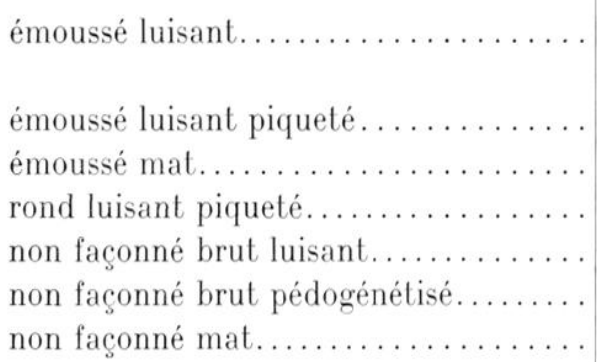 & \begin{tabular}{r|r}
12 & \\
9 & 21 \\
15 & \\
1 & \\
37 & \\
1 & 63 \\
25 & 6
\end{tabular} & $\begin{array}{r}21 \\
17 \\
14 \\
\left.\begin{array}{r}4 \\
27 \\
0 \\
17\end{array}\right\} 44\end{array}$ & \begin{tabular}{r|r}
6 & \\
24 & 30 \\
2 & \\
6 & \\
36 & \\
0 & 62 \\
26 &
\end{tabular} \\
\hline pourcentages.. & $100 \%$ & $100 \%$ & $100 \%$ \\
\hline
\end{tabular}

Le caractere alluvial est nettement plus marqué que dans le sol naturel. Les limons sont plus sableux ot ne possedent pas de matrice argileuse. Les trois couches sont nettement distinctes et sont formées d'apports latéraux par ruissellement et colluvionnement. surtout le limon jaune qui recoure le sol areheologique. Le ruissellement semble en rapport direct aver la légere pente de la terrasse de Sainte-Agalhe, qui est inclinée vers le nord-ouest avec une pente d'environ $1 \mathrm{~m}$ pour $100 \mathrm{~m}$. Ies éléments éoliens sont en régression par rapport aux éléments fluviatiles et ont raisemblablement pour origine les remplissages plus anciens (Würm) érodes par les ruissellements.

Lin déapage horizontal, le sol archéologique correspond à une aire de piétinement, constitué de plusieurs lentilles, d'une épaisseur totale d'environ $10 \mathrm{~cm}$. Huit curettes creusées dans le sol naturel s'intercalent dans les différents niveaux de piétinement (fig. 3 at 1) : deux cuvettes (arres EF 11 et F 17) ovoüdes de $80 \mathrm{~cm}$ sur 50 cm environ, pour 15 ) cm de profondeur moyenne, posident un remplissige tres charbonneux. Ce sont vraisemblablement des foyers; six cuvettes ovoüdes, plus ou moins allongées, de mèmes dimensions moyennes, sont comblées par des colluvions et par de nombreux débris archéologiques. Ieur utilisation reste hypothétique : l'absence d'éléments carbonisés permet d'éliminer une utilisation an foyer; il est possible qu'elles soient de simples cuvettes

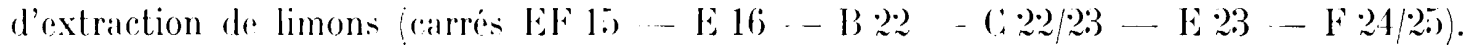


Dans le carré F 16 , en limite de notre décapage, apparaît un trou de poteau quadrangulaire, de $20 \mathrm{~cm}$ de côté et de $25 \mathrm{~cm}$ de profondeur. Cette implantation de poteau est isolée : si elle appartient à une structure d'habitat, il faudrait chercher celle-ci dans la partie nord du chanticr.

Sur les sols, sont répartis de très nombreux galets éclatés par le feu, fragments d'argile cuite ou vitrifiée (scories) et de minuscules tessons. Tessons et fragments d'argile cuite sont roulés et arrondis par les piétinements. Ces débris archéologiques ne sont pas dispersés de façon uniforme sur les $70 \mathrm{~m}^{2}$ fouillés (fig. 3 et 4) : il y a concentration du matériel au nord de la fouille, et dispersion vers l'est, le sud et l'ouest; la zone nord de concentration du matéricl correspond à une zone subhorizontale. De nombreux éléments sont groupés au fond des cuvettes du sol. Dans les secteurs pauvres, le sol est légèrement incliné (pente d'environ $2 \mathrm{~cm}$ par mètre). Deux interprétations sont possibles : ou l'occupation humaine a été limitée à la zone horizontale (nord du chantier), ou des lessivages des zones pentues ont entraîné le mobilier dans les parties basses horizontales ou dans les cuvettes.

Le mobilier archéologique recueilli dans le sol d'habitat n'a pas pu faire l'objet de déterminations précises, du fait de sa fragmentation.

\section{LA Fossl: $x^{0} 1$.}

Située dans la zone ouest du chantier (fig. 3), d'une profondeur totale de $1 \mathrm{~m}$, elle possède des parois surplombantes : son diamètre est de $1,5,5 \mathrm{~m}$ au sommet, do $1,90 \mathrm{~m}$ à la base (fig. כ).

En stratigraphie, nous distinguons de bas en haut neuf niveaux archéologicques répartis en trois ensembles.

Ensemble inferieur. Niveau IX b : au fond de la fosse, le sol limoneux présente des fentes de rétraction qui lui confèrent l'aspect d'un sol polygonal et sont comblées par des inclusions blanchitres dues aux lessivages postérieurs. Sur ce sol subsistent des traces de foyer. arec cendres et débris charbonneux. I la partie supérieure du foyer sont concentrés de nombreux fragments d'argile cuite, des galets éclatés, des tessons, des morceaux de gries srûlés et des inclusions d'argile jaune. L'ensemble des vestiges est noyé dans une matrice brune et grasse;

niveau IX a : il est composé en grande parlie de plaquetles calcaires et gréseuses calcinées, de "briques" en argile cuile; les interstices sont comblés par des galets et des gravillons liés par une argile cuite de couleur grise. Le tout repose sur une couche de cendres el de charbons de bois;

niveau VIII : il est disposé en lentilles biconcaves : au centre, un niveau charbonneux d'une dizaine de centimetres d'épaisseur devient plus noir à la base; de part et d'autre, un remplissage argileux gris-blane, d'une épaisseur totale de 40 i $5 \mathrm{~cm}$, contient des inclusions d'argile jaune plus ou moins cuite, des charbons et des tessons.

Les niveaux $I X$ a el $I X$ b ont une surface convexe. Le niveau VIII assure la transition avec les niveaux supérieurs. La partie centrale et superficielle du niveau VIII, de forme concave, parait légèrement remaniée : aux remplissages argileux sont mêlés des tessons et des fragments d'argile cuite; les strates horizontales sont recoupées par le niveau VII.

Ensemble moyen. Niveau VII : d'une épaisseur totale de 1:5 à $20 \mathrm{~cm}$, il est constitué de limons bruns foncés, plus ou moins sombres. Des inclusions d'argile cuite ou crue de couleur gris jaune et des charbons de bois sont plus denses dans la partie orientale du niveau : des lentilles d'argile 


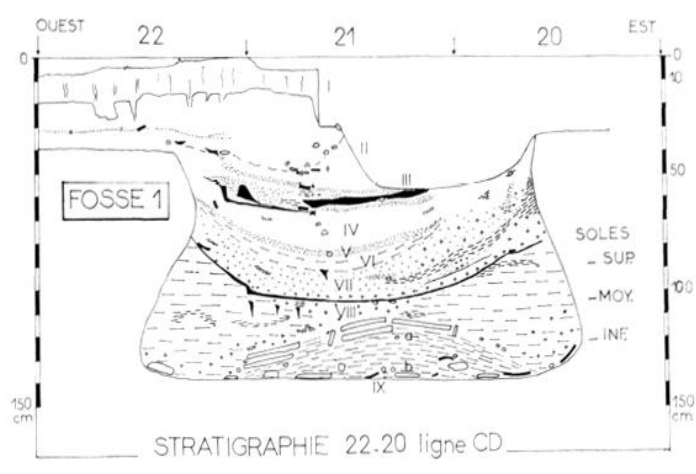

5) Fosse no 1. Slratioraphie lransversale.

6 La fosse $n^{\circ}$ l est grossierement circulaire, a fond plat et parois surplombantes. In can calisation on tranchée a coupe la fosse suivant son axe. $\rightarrow$

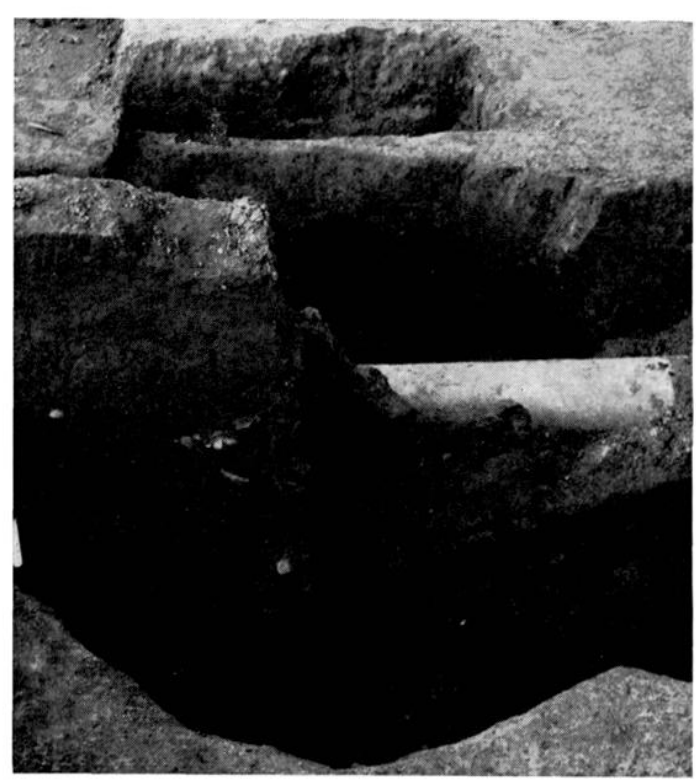

7 l.es remplissages de la fosse $n^{0} 1$ : all sommel, les niveaux supérieurs limoneux; en dessous, les couches moỵennes d'argile cuite, régulierement disposées en lentilles.
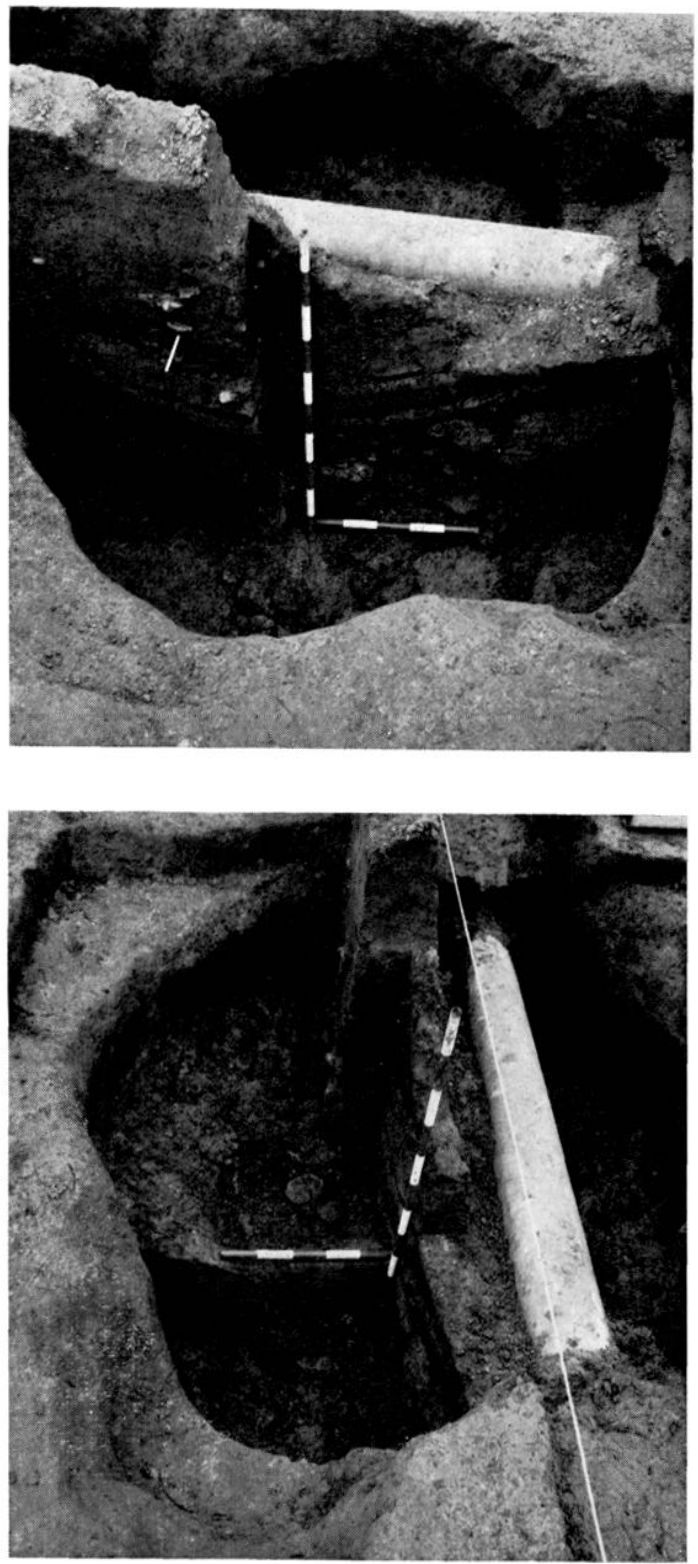

$x$ Structures de la fosse no 1 . Galets et plaquettes calcaires sont groupés sur les foyers au centre de la fosse. Fragments d'argile cuite et tessons sont plus nombreux en bordure de la fosse.

gris-jaune sont interstratifiées entre des niveaux charbonneux. A la base, un niveau de charbons sépare nettement le niveau VII de l'ensemble des couches inférieures;

niveau VI : arguile cuite, sèche et dure, de couleur gris-blanc, a une puissance moyenne de 4 a $8 \mathrm{~cm}$. Il se termine en biseau vers l'est;

niveau IV : des limons gris-jaunes de 10 a $20 \mathrm{~cm}$ d'épaisseur moyenne contiennent de rares inclusions de galets et d'argile cuite.

Les niveaux VII à IV sont disposés en cuvettes régulièrement stratifiées ; les fragments d'argile cuite sont concentrés surtout en bordure de ces cuvettes, au contact des parois de la fosse. 
Ensemble supérieur. Niveau III : des lentilles d'argile cuite, rouge, allernent, aver des courhes charbonneuses. Elles ne contiennent que quelques tessons et inclusions d'argile jaune. lópaissur tolale atteint une dizaine de centimotres;

niveau II : disposé en légere ruvette, son épaisseur varie de 20 a :35 (am. Il est formé d'argile gris-jaune. A la partie inférieure, quelques charbons épars, tessons, galets et fragment.s d'argile ruite soulignent un sol;

niveau I : il s'agit du sol superficiel actuel, d'une epaisseur movenne de $15 \mathrm{~cm}$. Des aroiles sableuses, de couleur gris-jaune, sont perturbées par les labours el par les animaux fouisseurs.

Les niveaux III a I sont disposes horizonlalement ou en légre cuvetle.

Etablissement de la fosse. La fosse $\mathrm{n}^{0} 1$, profonde de 1,10 m est creusée dans les limons en place, présente une forme particulière. En plan, elle est approximativement circulaire. Son diametre à la base est de $1,7 \mathrm{~m}$. A la partic supérieure, ses parois se rapprochent progressivement. A l'ouverture, le diamètre de la fosse n'est plus que de $1, \overline{5} \mathrm{~m}$, d'où un profil général en eteignoir. le fond de la fosse est plat et a subi l'action de la chaleur, comme en témoignent les fentes de rétraction en structure polygonale.

En décrivant la stratigraphie, nous avons distingué trois morles de remplissage : convexes a la base, en cuvettes concentriques, lenticulaires au sommet.

Struclures inférieures (nireaux $I X$ à $V$ ). Les remplissages sont en majorité composés de niveaux d'argile cuite superposés; les charbons et les éléments qui y sont mêlés ont tous éte calcinés (galets, scories argileuses, tessons tres poreux, plaquettes de calcaire ou de grese, etc.). La profonde calcination, voir la vitrification partielle de la paroi dans les deux tiers inférirurs de la fosse témoigne d'un feu arrent.

Lne première utilisation peut être mise en evidence an fond de la fosse, directement au contact de l'argile en place. Les plaquettes calcaires et gréseuses sont concentrées surtout vers le centre.

Une seconde ulilisation est bien matérialisée par le niveau $I X$ a (sole inférieure de la fig. 6). Ln aménagement soigné a été réalisé : une chape d'argile a recouvert les vestiges de la première utilisation, recouverte elle-même de plaquettes de calcaire de dimension variant entre 10 of $50 \mathrm{~cm}$ de côté, disposées sur une ou deux épaisseurs et auxquelles s'ajoutent des dallettes de grese, des fragments de granit calcinés (meules ?), ainsi que des cailloutis. Lne couche d'argile cuite recoure le tout; on y rencontre des "briques" à surface lissée.

Le décapage de la sole inférieure (fig. 8 et 9) met en évidence deux zones concentriques distinctes : au centre, un foyer a été établi directement sur la structure décrite ci-dessus. Les tessons de céramique, sans ètre absents, y sont peu nombreux; -- à la périphérie, une couronne d'argile cuite correspondant au fond de la fosse (figr. 5), done en dépression, a fourni de nombreux tessons souvent calcinés et poreux. Des "supports de cuisson" (?) en argile cuite de section triangulaire aux angles arrondis (longueur : $10 \mathrm{~cm}$, largeur : $7 \mathrm{~cm}$, épaisseur : $8 \mathrm{~cm}$ ) sont répartis irrégulièrement dans les $20 \mathrm{~cm}$ de borlure du foyer.

Deux utilisations ont été mises en évidence : sole inférieure (fig. 9), sole moyenne (fig. 9). Lat sole inférieure ayant été conservée probablement dans son état ancien, un remplissage argileux hétérogène a été ajouté sur le pourtour du foyer alin de combler 

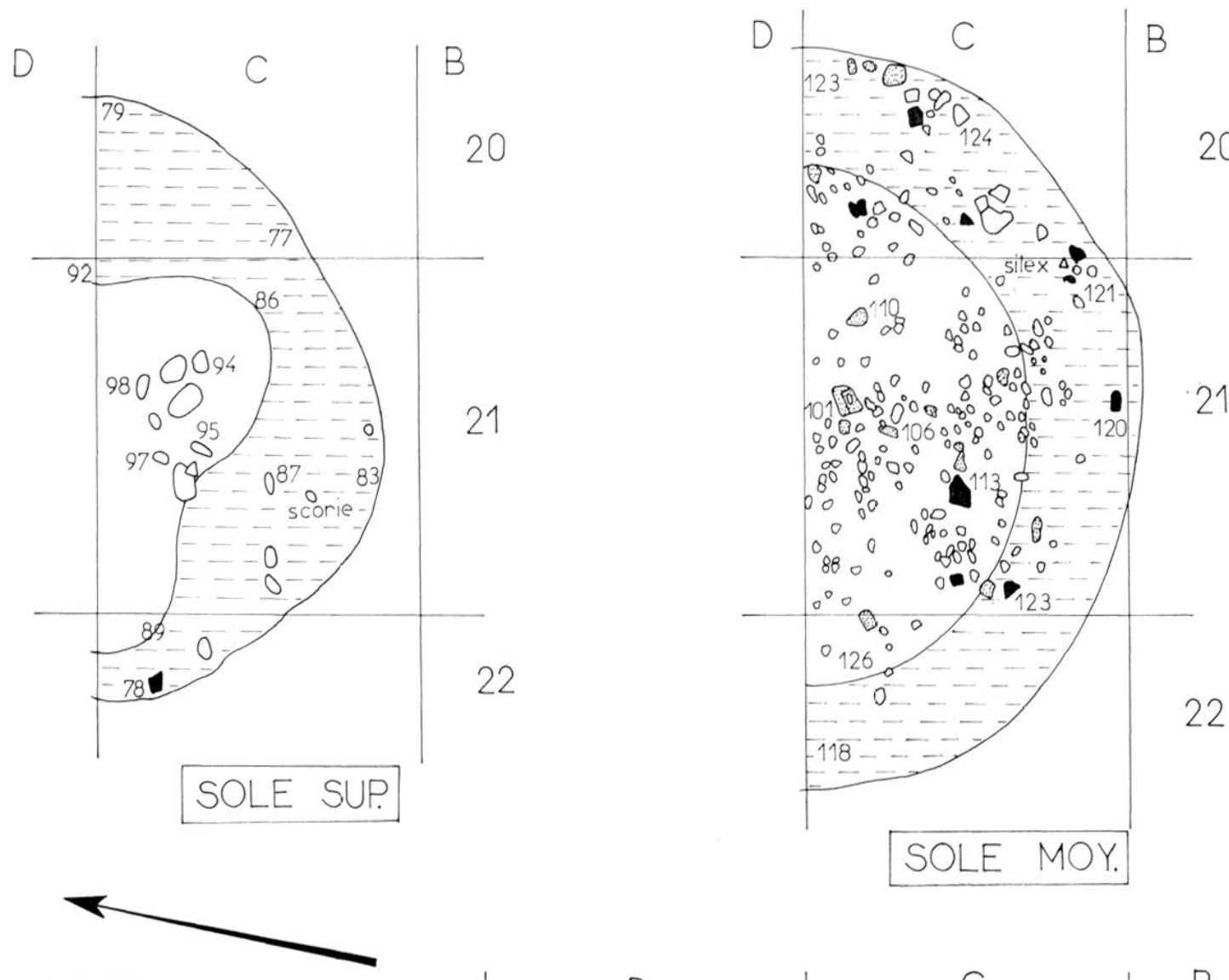

NORD magn.
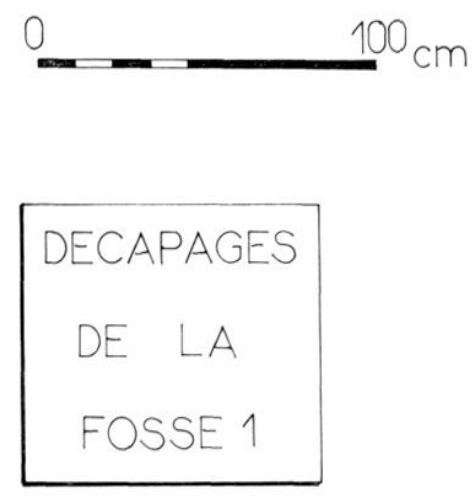

Aragment de poterie

( " d'argile cuite

- galer siliceux

If dalle calcaire

$\Rightarrow$ aire d'argile cuite

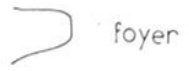

79 core de profondeur

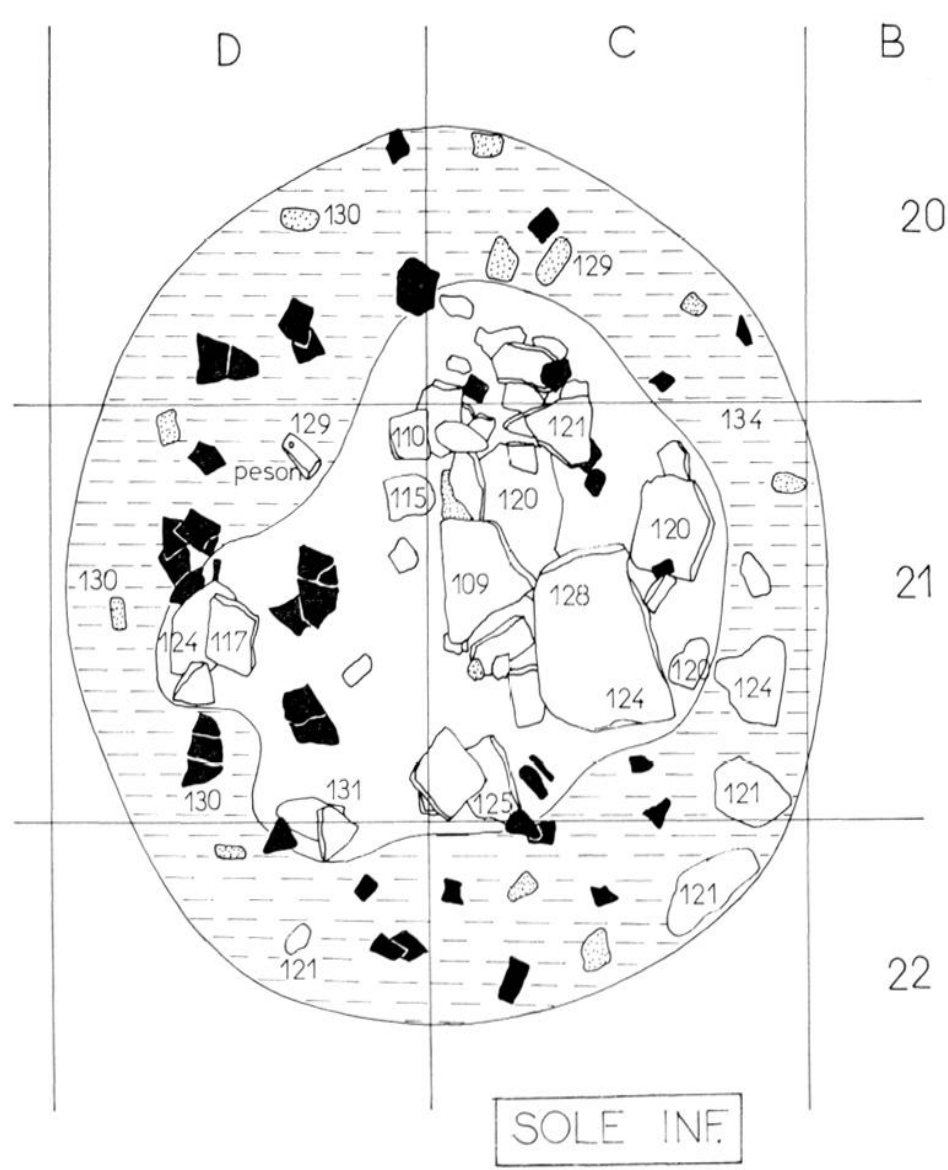

9 Fosse no 1 . Sur le pourtour, banquet te daropile rapportie. Amenagument de galets dans la partie centrale en curetle. la sole inferieure apparait an fond du sondage. 
la dépression le long de la paroi. La fosse ainsi transformée présente une sorte de banquette tout autour du foyer installé dans un creux cette fois-ci, et reposant sur des galets (couche VIII et sole supérieure, fig. 9).

Struclures moyennes (niveaux IV à VII). Les niveaux sont composés d'une succession de couches d'argile calcinées dans leur partic supéricure, venant s'empiler en cuvettes. Les éléments charbonneux y sont rares, les galets peu nombreux sont présents dans le remplissage argileux, mais on ne retrouve plus les dispositions observées dans les couches VIII et IX. Ces niveaux donnent l'impression d'une succession d'aménagements semblables au foyer de la sole supéricure (fig. 9). Après chaque cuisson, l'aire d'argile a été nettoyée des vestiges de foyer et une nouvelle couche d'argile à nouveau disposée.

Structures supérieures (niveaux II et III). Avec ses successions d'argile cuite et de lentilles charbonneuses, la structure du niveau III est encore différente de l'ensemble moyen et inférieur. Peut-être correspond-il à un réemploi tardif et peu important sans aucun souci de nettoyage. Avec ses galets dispersés mêlés aux tessons et aux fragments d'argile cuite, le niveau II semble résulter d'un remblaiement de la fosse.

\section{Mobilier archéologique.}

Tous les éléments du mobilier sont cotés et relevés niveau par niveau. Comme il ne semble pas y avoir d'évolution du mobilier en rapport avec la stratigraphic, nous présentons ici les pièces en les regroupant suivant les trois grands ensembles stratigraphiques.

Ensemble inférieur. La céramique est très fragmentée et tous les tessons ont subi une cuisson excessive, quelquefois même une vitrification partielle. Formes et décors sont peu variés : urnes ou gobelets à bord rentrant (fig. 13, $\mathrm{n}^{0} 9$ ), droit (fig. 13, $\mathrm{n}^{0} 10$ ) ou légèrement déversé (fig. 13, n० 7) ; urnes carénées (fig. 13, n 3) ; bols à bords rentrant (fig. 14, $\mathrm{n}^{\circ} 2$ et 1); urne à cordon horizontal impressionné au doigt (fig. 13, no 6).

De grosses pièces en terre cuite proviennent du même ensemble: peson à perforation horizontale (fig. 13, no 12) ; fragments d'argile vitrifiée ou scoriacée ; "briques" à forme de parallélépipèdes droits à angles arrondis, leur surface étant simplement lissée à la main (longueur : $14 \mathrm{~cm}$, section : $6 \times 6 \mathrm{~cm}$ environ).

Les galets et les plaquettes déposés sur les foyers ont été examinés par ‥ N. Théobald (Faculté des Sciences, Besançon). Ils ont des origines différentes : les galets de granite très altéré et les galets de quartzite sont des éléments alluviaux de la vallée de la Moselle ; des grès micacés très fins à ciment siliceux (Trias) ressemblent aux grès à Voltzia ; les grès très fins à ciment calcaire sont semblables aux grès du Rhétien; un calcaire très détritique, après attaque à l'acide chlorhydrique, laisse entrevoir des éléments calcaires et les restes d'une trame siliceuse.

Galets et plaquettes peuvent se trouver dans un rayon d'environ $2 \mathrm{~km}$ du gisement de Sainte-Agathe, mais ils n'existent pas à l'état naturel dans le gisement même. Leur apport est donc un fait humain volontaire.

Ensemble moyen. Nous avons relevé à ce niveau cinq fragments de silex taillé : une plaquette quadrangulaire à retouches marginales à une extrémité (fig. 10, no 1); un éclat laminaire brut (fig. $10, n^{0} 2$ ); un éclat correspondant à un réaménagement de nucléus 

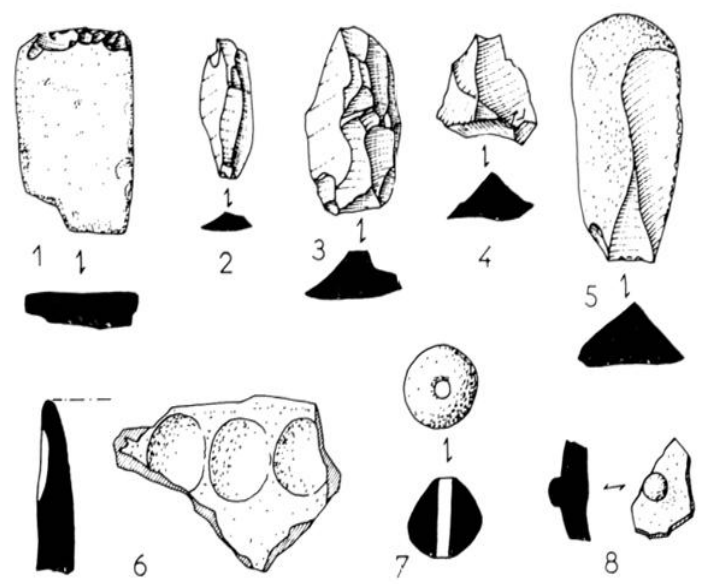

\section{FOSSE 1}

I0 Mobilier de la fosse $n^{n} 1.1$ : grattoir sur placquelte de silex (niv. moyen . 3-4 : celats nir. moyen . 5) : coutteau à dos naturel cortex portant des retouches diutilisation niv. moyen . 6 : rebord à decors d'impressions digitales deblais: 7 : fusalole on grain d'onfilage déblais. $x$ : element de decor pastilli deblai;:
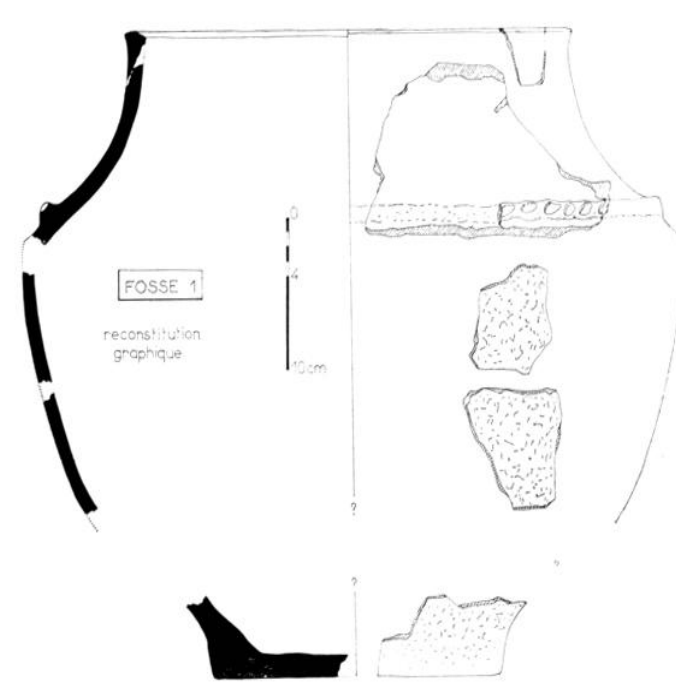

11 lrne carené à bord renlrant et à cordon impressionné sur la carrine 'nis. supérieur.

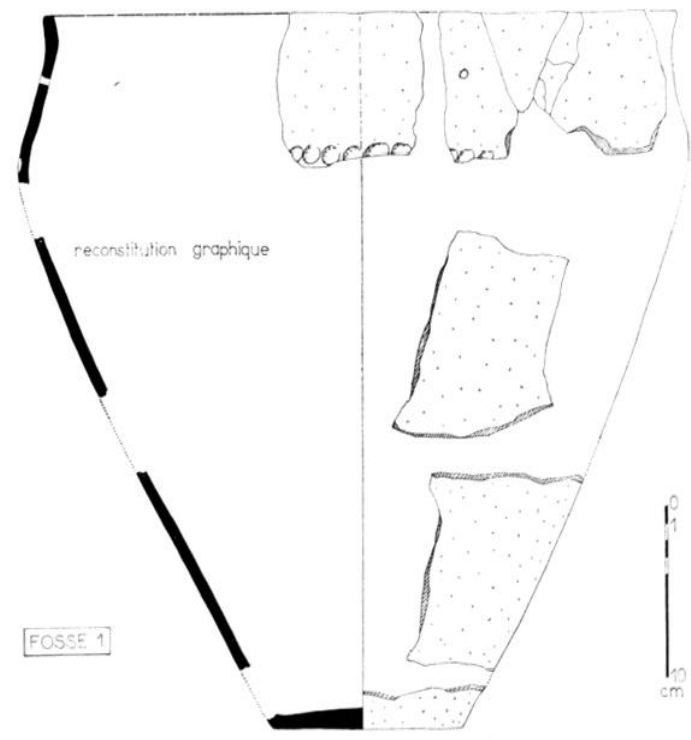

12 Line à décor impressionné au doigh (nivean moỵne

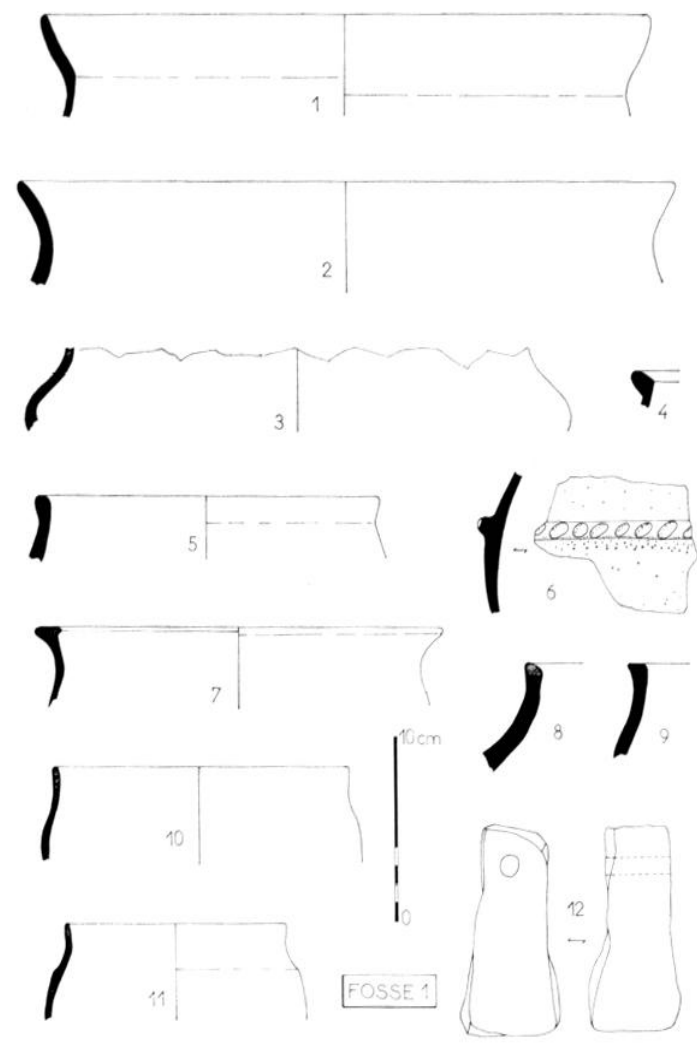

13 1, 2, 5, 11 : rebords diurnes des niveaux movens. $3,4,7,9,10$ : rebords d'urnes des niveaux inférieurs. 8 : rebord d'urne déblais . 6 : decors en cordon a impression digitale niveau inférieur, 12: peson en argile cuite niveau inférieur.
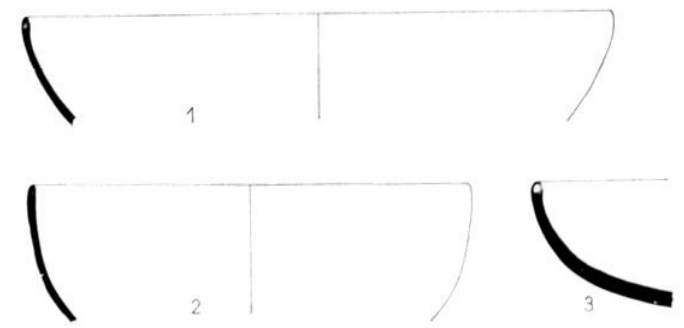

(
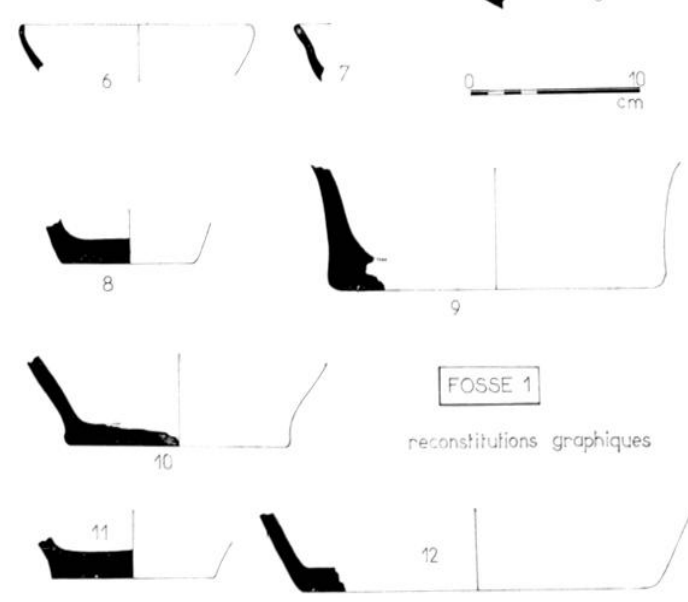

141 et $2:$ bols (niveau inférieur). $3,4,7:$ bols niveau moven! 8,11 : fonds plats déblais . 9, 10 : fonds plats (niveau superieur. 12: fond plat (niveau moyen,. 


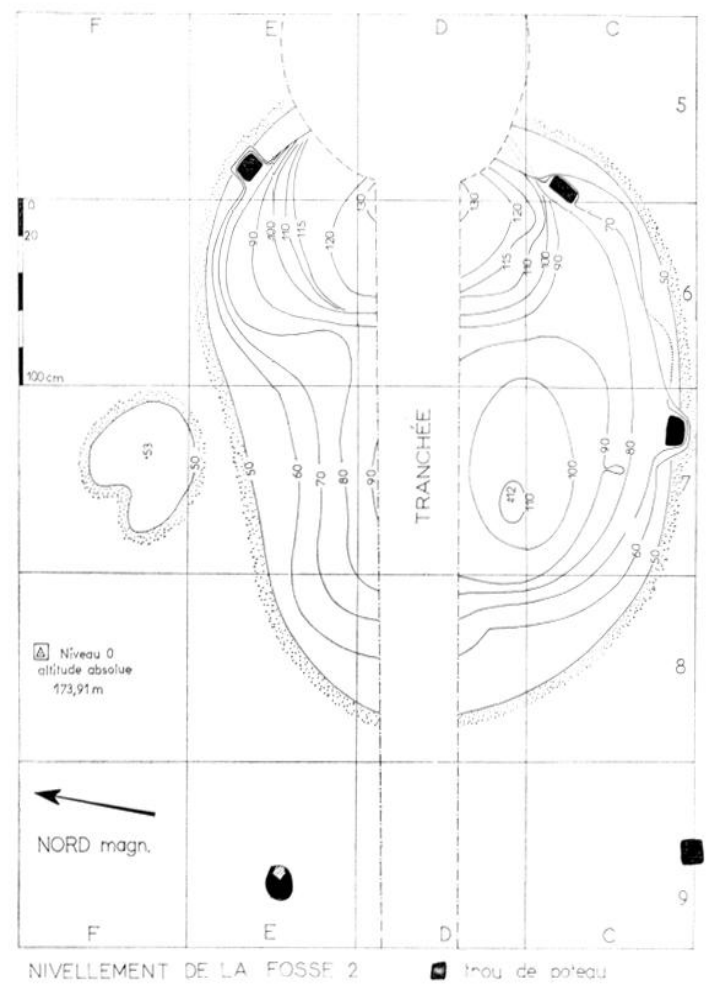

15 Fosse no 2. Nivellement de la fosse of struclures ercadranl celle-ei.

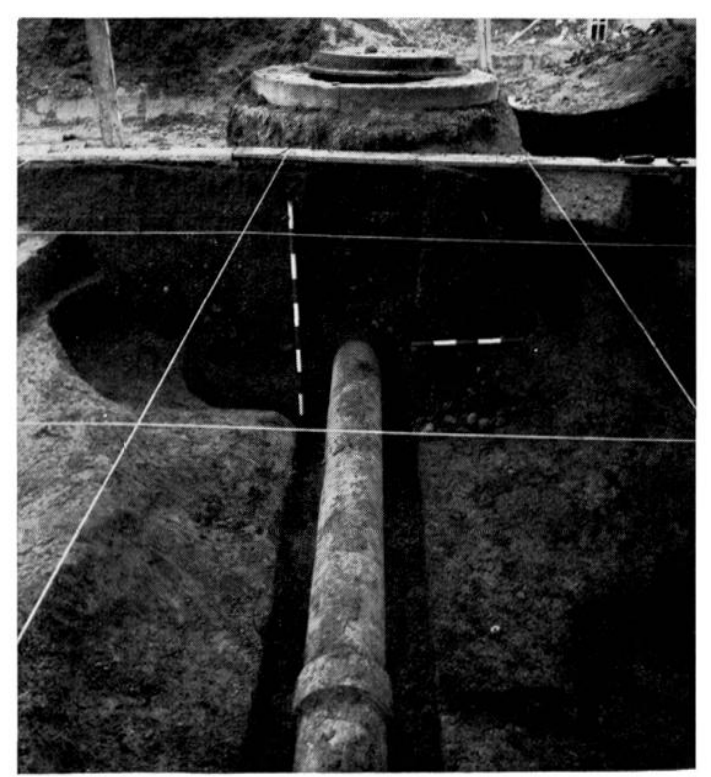

16 Structure en gradins de la fosse no 2 , recoupés par la canalisation et en parlit detruile par un regard.

prismatique (fig. 10, no 3); un erlat brut a enlevements convergents fig. 10, no 1 ) ; un éclat laminaire à dos cortical et retouches d'utilisation (fig. 10, no $\mathrm{n}^{0}$ ).

La céramique présente les mèmes aractères que celle dr l'ensemble inférieur : urne à décor impressionné all doigt (fig. 1:2); urnes à rebord léversé (fig. 13, nos 1 et 2$)$ ou légèrement rentrant (fig. 13, nos j) ot 11); bols à bord rentrant (fig. 14, nos 33 et 4) ; jatte carénée à bord rléversé (fig. 14, no 7 ).

De nombreux fragments d'argile vitrifiée sont répartis dans ces niveaux. (ialets et plaquettes ont la mème origine que ceux de l'ensemble inférieur.

Ensemble supérieur. Les fragments de céramique sont rares : urne carénée à bord rentrant et cordon impresionne sur la carène (firg. 11) ; deux fonds plats qui appartiennent vraisemblablement à des urnes (tig. $14, n^{0 s}$ ? at 10 ).

Les fragments d'argile cuite et vitrifiee sont inexistants dans la partie supérieure des remplissages. De rares fragments osseux sont determinalles.

Déblais. Quelques pièces intéressantes ont été trouvées dans les déblais de la tranchée qui recoupe la fosse 1, ou dans les couches supérieures fouiliees par le Contr. Lormain de recherches humaines : une petite fusaïole ou grain d'enfilage en terre cuite (fig. 10, no 7); un tesson avec pastille collée sur la pâte (fig. 10, no 8) ; rebord avec décor à impressions digitales (fig. 4, no 10 ); fragments de bol (fig. 14, no (6) ot de fonds plats (fig. 14, nos 8 et 11). 
LA rosse $x^{0} \stackrel{2}{2}$

La fosse 2 , plus irrégulière, de :3 m de còté et profonde de 1,3 $\mathrm{m}$, est située à l'est de la première.

Les dépòts sont disposés dans une fosse à profil en gradins (fig. 17). Trois grands rnsembles stratigraphiques correspondent a l'extérieur de la fosse à deux sols de piétinement differents.

Ensemble inferieur. I'ne lentille de limons tris charbonneux de 1 is 8 cm d'épaisseur, situé sur la partie haute du fond de la fosie niveau VII b. lig. 18. est recouverte d'une couche de limons gris durcis par la ruison niveau VII. Celte courhe d'environ 30 com d'épaisseur conlient quelques inclusions dargile ruile, des charbons epars el quelques lessons. plus denses dans la parlie sud de lil fosise.

Einsemble moyen. De bats en haul. nous trouvons:

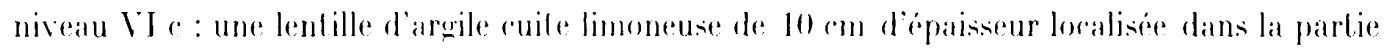
oucest de lit fosse;

niveau VI b : des limmo gris de i) it lis cm d'epaisseur contenant quelques lentilles d'argile ruile; des galets sonl présents dans la parlie orcidentale de la fossere;

niveaux VI a ct VI : un aggomérat de placques d'argile cuite et de galets éclatés an feu, qui peut atteindre 25) (com d'épaisseur dans la partie supérieure de la fosse.

Lensemble du niveau VI a une structure lenticulaire oblique, en còne de déjection. Le pendage des rouches vers l'esl, les nombreuses inclusions d'argile cuile, gralets, tessons, disposés anarchiquement, lui conferent un aspect remanié. Ces dépoits, enfin, sont localisés en bordure de la fosse et leur formal ion parait très rapide.

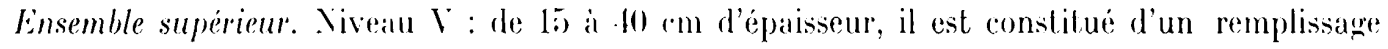
tress charbonneux, noiraitre. Iris dense a la parlie inférieure. Il contient de nombreux galets, fragments d'argile ruile et tessons: ;

niveau IV : un limon gris diune épaisseur de jo a 15 cm renferme quelques rharbons el fragments d'argile cuile noire au rouge. Les galets sont tolalement absents;

niveau III : de faible śpaisseur, 5) cm, il est composi d'une couche de limon gris tries charbonneux, renfermanl de rares galets et tessons;

niveau II : limoneux jaune, il comporte des particules d'argile avite, des pelits charbons de bois el des nodules de fer;

niveau I : sol superficiel actuel, argilo-sableux de leinte gris-jaune; on y retrouve des petils éléments roulés. Il a élé perturbé par les labours anciens et les lerriers d'animaux;

niveau 0 : déblais des fouilles anterieures et des lravaux de construction du lotissement.

L'ensemble des couches supérieures occupe loute la surface de la fosse. La disposition est souvent horizontale ou en légrere dépression.

Cette fosse, dont la bordure orientale est détruite par une bouche d'égout, apparait comme une succesion de cureltes emboitées descendant en gradins d'est en ouest, de 0,50 a $1,30 \mathrm{~m}$ de profondeur (fig. 15). Ces gradins sont également visibles suivant une coupe nord-sud (fig. 16 et 17 ). Trois cuvettes sont distinctes.

Cing trous de poteaux $\left(\mathrm{E}_{5}, \mathrm{C}_{5}, \mathrm{C}_{7}, \mathrm{E}_{9}, \mathrm{C}_{9}\right)$ a section quadrangulaire ou arrondie avec pierres de calage soulignent le pourtour de la fosse (fig. 15). L'aménagement de ces pieux 
est contemporain du creusement de la fosse 2 (sol b) ; le sol a, consécutif à la période de réemploi, les recouvrait (fig. 17).

Le niveau supérieur de la fosse 2 (fig. 15 et 18 ) est recouvert d'une zone charbonneuse dont la bordure extérieure comprend une cuvette de $3 \mathrm{~cm}$ de profondeur pour un diamètre moyen de $60 \mathrm{~cm}$ (fig. 15 et 7 ). L'existence d'un trou de poteau en cet endroit est vraisemblable ; malheureusement, les fouilles antérieures ont traversé le niveau archéologique. Si l'on admet que les trous de poteaux délimitent un enclos quadrangulaire autour de la fosse 2 , la cuvette F7 serait symétrique du poteau $\mathrm{C7}$.

Struclure inférieure. A la base des remplissages, une cuvette possède en pourtour une banquette d'argile cuite (fig. 18). Le fond de la cuvette n'est pas cuit, mais présente des fentes de rétraction qui lui donnent un aspect de sol polygonal. La cuvette est comblée par le dépôt régulier et homogène de la couche VI (fig. 17).

Structure moyenne. Son remplissage hétérogène, d'aspect lenticulaire, est à léger pendage oblique. Cette disposition se reflète dans celle des objets contenus dans le remplissage. Les galets, les tessons qui reposent souvent sur la tranche, les morceaux d'argile cuite à surface lissée suivent un léger pendage vers le sud-est de la fosse. Ce matériel est plus abondant dans la partie sud-est, la plus profonde, en $C_{5}$, $C_{66}$ et $C_{7}$ (fig. 15), 17, 19 et 20). Au contraire, les objets sont en plus faible densité dans la partie nord-est de la fosse, à faible pendage, en $\mathrm{E}_{6}$ et $\mathrm{E}_{8}$ (fig. $15,16,19$ et 20). Des tessons d'assez grosses dimensions, découverts au fond de la fosse, dans sa partie sud-est, se raccordent avec d'autres fragments plus petits découverts dans la partie nord-est. Cette céramique garnit essentiellement le bord nord de la fosse.

La concentration du mobilier est maximale dans la partie profonde de la fosse et régresse dans sa partie la plus élevée (fig. 19 et 20 ). La dispersion des objets à l'intérieur de la fosse paraît se faire à partir du carré $E_{7}$ et selon le pendage. L'aspect qui s'en dégage est celui d'un cône de déjection.

Structure supérieure. Les niveaux sont en dépôt subhorizontal. Un foyer d'habitat est séparé de la structure moyenne par une couche quasi-stérile; il occupe presque toute la surface de la fosse. Ces niveaux paraissent correspondre à un habitat temporaire et à un remblaiement naturel de la fosse par colluvionnement.

\section{Le mobilier archéologique.}

Ensemble inférieur. Le mobilier des niveaux VII et VII b est très paure: des scories et boulettes d'argile vitrifiée, des tessons très fragmentés ayant subi une forte cuisson ne peuvent donner lieu à une détermination précise. Deux objets en bronze proviennent de la surface du niveau VII : bracelet à jonc torique, décoré de dix groupes d'incisions transversales (fig. 22, $n^{0} 2$ ) ; aiguille à chas brisé (fig. $22, n^{0} 3$ ).

Ensemble moyen. La quasi-totalité des objets a été recueillie dans les niveaux VI et V. Trois éclats de silex attestent une industrie lithique tardive : grattoir latéral sur éclat à dos réservé (fig. $21, \mathrm{n}^{0} 8$ ) ; éclat à enlèvements convergents et cortex particllement conservé (fig. 21, n 9) ; grattoir convexe sur éclat (fig. 21, $\mathrm{n}^{0} 10$ ). 

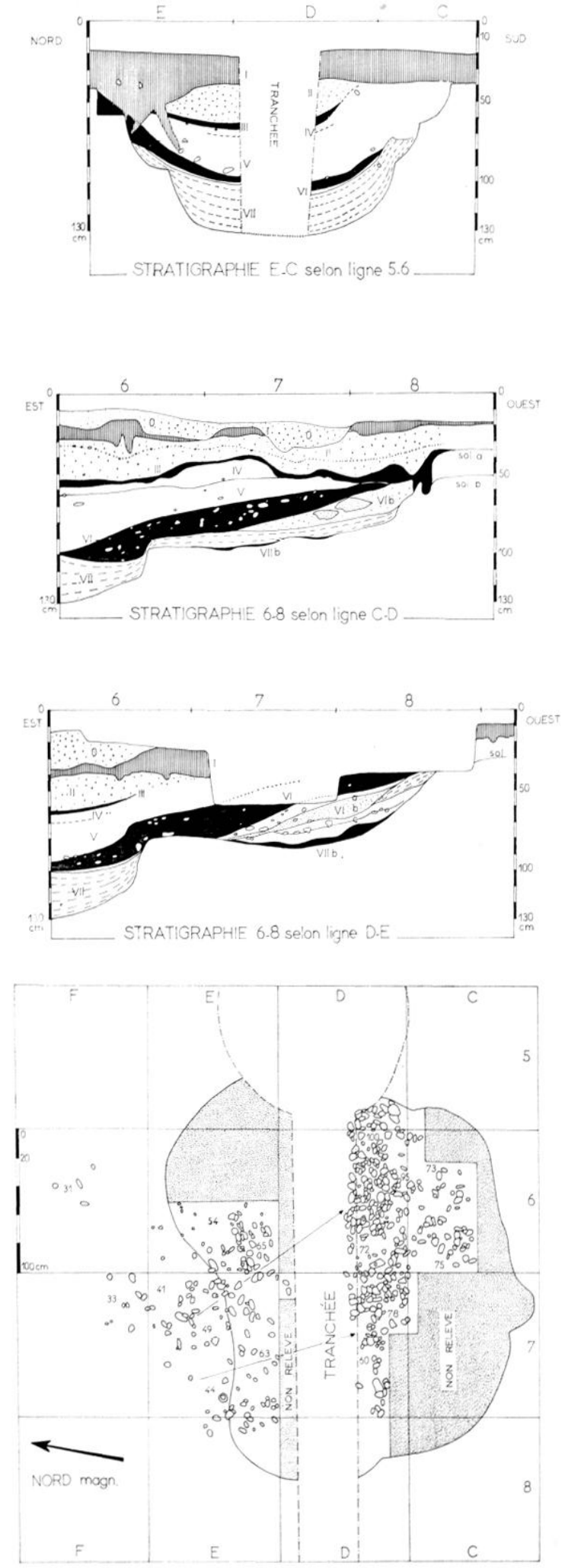

NIVEAU SUPERIEUR DE LA FOSSE 2

répartition des galets

19 Niveau supérieur de la fosse no 2. L'accumulation des galets (éléments densesi dans les parties basses de la fosse et le pendage nord-nord-onest, sud-sud-est de leur répartition révèle l'existence de cònes de déjection, déjà mise en évidence sur la figure 18.

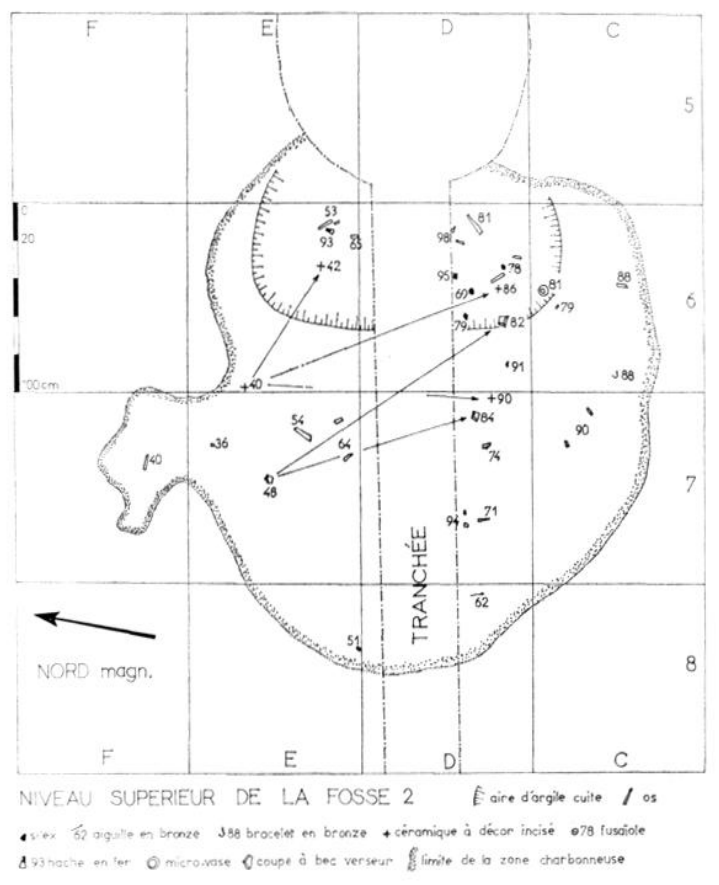

18 Niveau supérieur de la fosse no 2 . Répartition spatiale des vestiges archéologiques caractéristiques. l.es fleches indiquent. la dispersion des tessons d'une même céramique selon un ou plusieurs cônes de déjection ayant pour origine la partie nord de la fosse.

17 Stratigraphie transversale et longitudinale de la $\leftarrow$ fosse $n^{0} 2$.

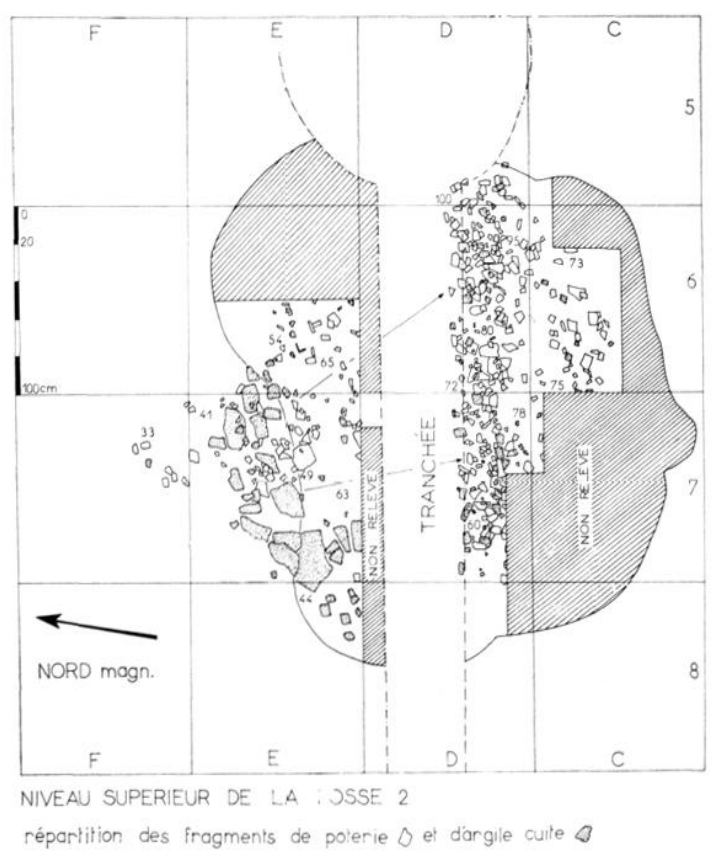

20 Niveau supérieur de la fosse 2. Répartition des fragments de poterie el d'argile cuite à l'intérieur de la fosse. Ievur accumulation dans les parties les plus basses de celle-ci. 

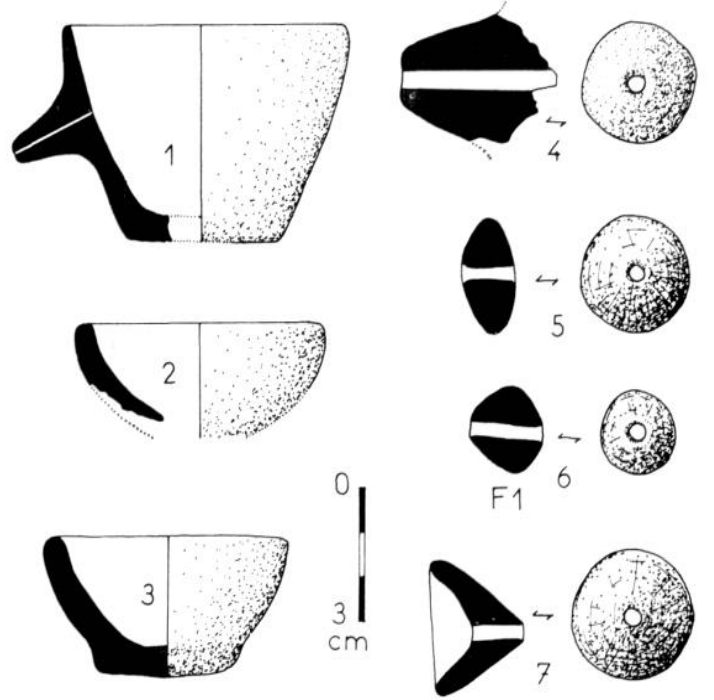

\section{FOSSE 2}
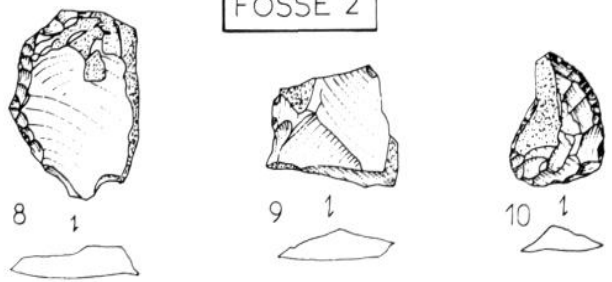

21 Fosse no 2 . 1 el 4 : vase biberon. 2 et 3: microvases. 5 a 7 : fusaioles ou grains d'enfilage. $x$ at 10 : grattoirs. 9 : ecclat.

221 : herminette en fer à ailerons terminaux. 2 : bracelet de bronze à molifs incisés. 3 : aiguille a chas en bronze. 1 : résidu de coulee ffer,

23 I a 7 : céramique peignere. Ceramique a decor incisé. Tessons à impressions digitales.
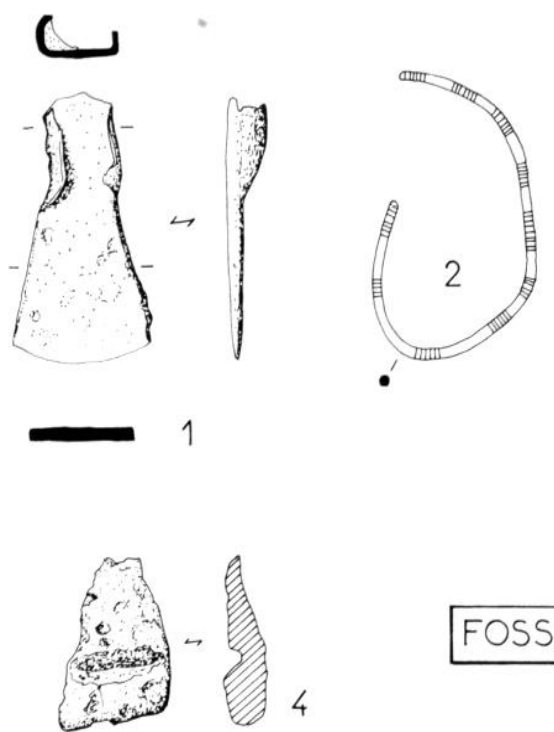

\section{FOSSE 2}

4

22
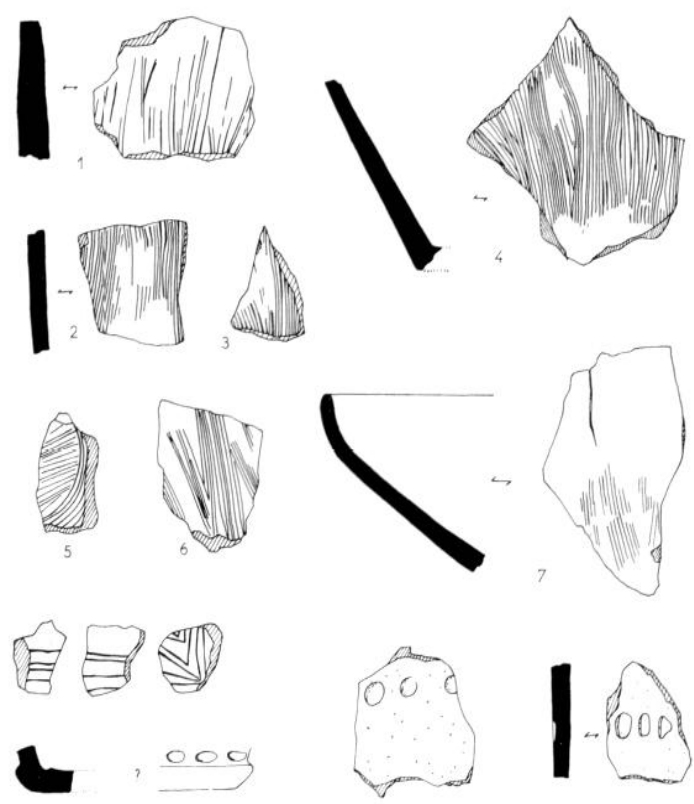

FOSSE 2

Do unême niveau proviennent deux éléments métalliques : herminette en fer, à légers ailerons terminaux (fig. $22, n^{0} 1$ ); fragment de coulée de fer brute (fir. $222, n^{0} 4$ ).

Deux fragments de meule dormante en grès (fig. $26, n^{0 s} 8$ ot 11) itaient situés parmi les galets éclatés par le feu, dans la rouche VI.

La céramique, souvent très cuite, légère et poreuse, peut ètre classée en dix catégories, en fonction des formes : coupe à indentations (fig. 24); microvases (fig. 21, nos 2 et 3); vases biberons (fig. $21, n^{\text {os }} 1$ et 4 ) ; urnes ou bols à décor peigné (fig. 23. nos 1 à 7 ) ; fragments d'urnes à décor incisé (fig. 23, en bas à gauche) ou impresionné au doigt (fig. 23, en bas 

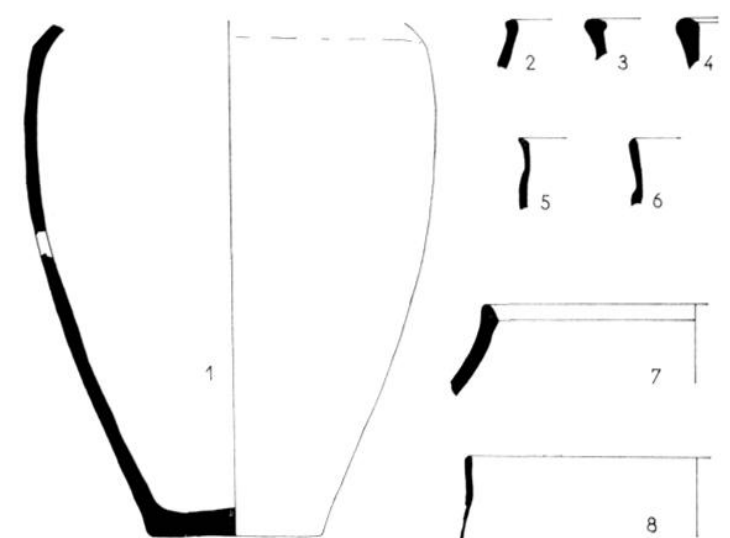

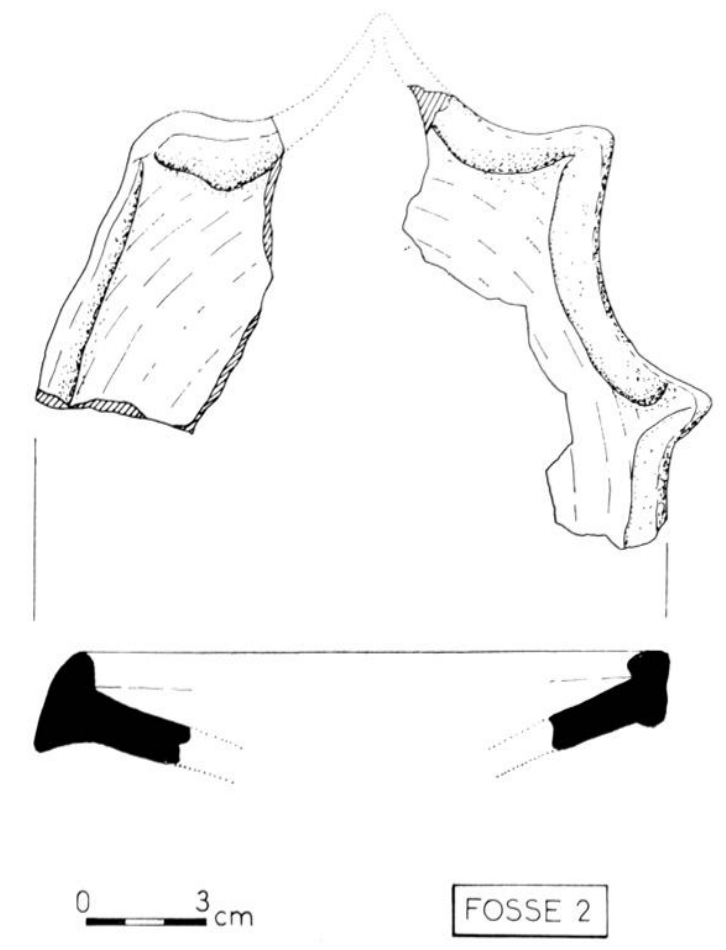

2.1 Coupe a indentations.
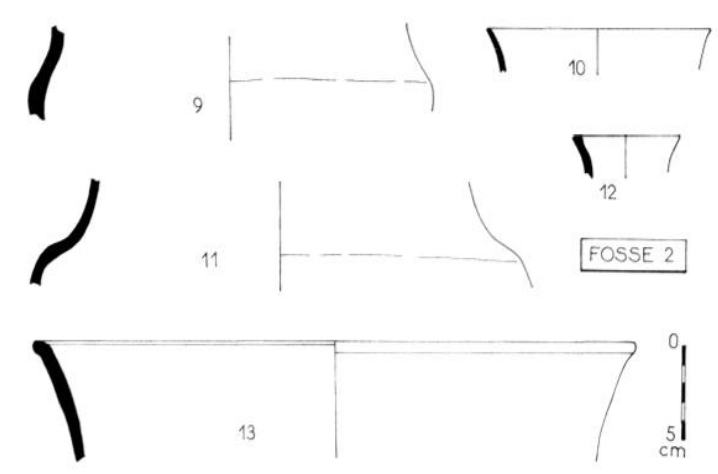

25) Urnes de la fosse no:2.

à droite) ; urnes carénées à bord rentrant (fig. 25, nos 1, 7, 9 et 11), droit (fig. 25, nos 5 et 6) ou déversé (fig. 25), nos 10 à 13); assiette à bord biseauté (fig. 26, no 2); fonds d'urnes ou de bols (fig. 26) ; jattes carénées à bord déversé (fig. $26, n^{\text {os }} 9$ et 10); bols (fig. 27 et 28).

Trois fusaïoles ou grains d'enfilage en terre cuite appartiennent au niveau VI (fig. 29 , nos ว̀ à 7$)$.

Quelques tessons ont été recueillis dans les déblais ou trouvés lors des fouilles du C.L.R.II. sans localisation valable. Des raccords faits avec des tessons localisés en stratigraphie nous permettent d'affirmer que tous ces éléments proviennent bien des mêmes niveaux.

Plaquettes et galets éclatés par le feu, fragments d'argile vitrifiée et masses d'argile cuite sont abondants dans les lentilles de l'ensemble moyen, les ossements animaux sont très rares.

Ensemble supérieur. Dans les foyers supéricurs, les céramiques sont très fragmentées et ne permettent guère de détermination spécifique. Les ossements animaux, brisés et calcinés, sont plus nombreux; porc-sanglier, ovicaprinés et bovidés. Plaques d'argile cuite et galets éclatés par le feu sont inexistants. 

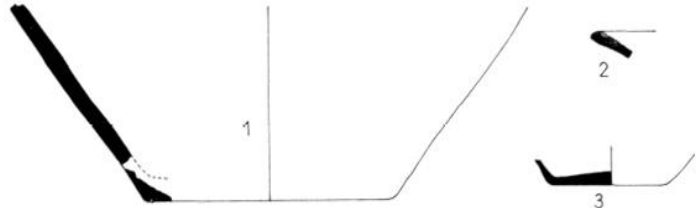

\section{FOSSE 2}
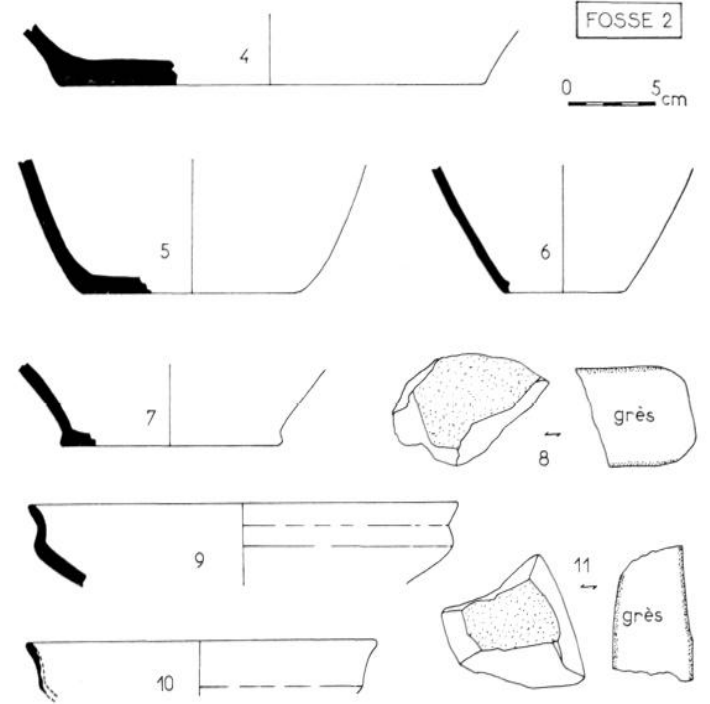

$261,3,5,6,7:$ fonds d'urnes oul de bols. $2:$ rebord d'assiette. 9 et 10 : jattes carénées. 8 et 11 : fragments de meules dormantes en gres.
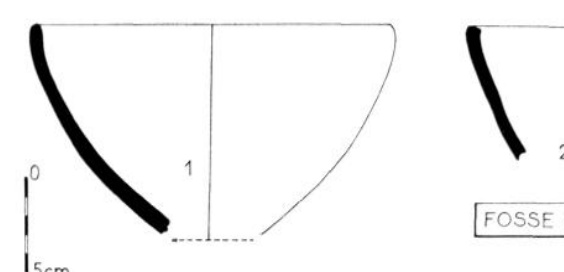

FOSSE 2
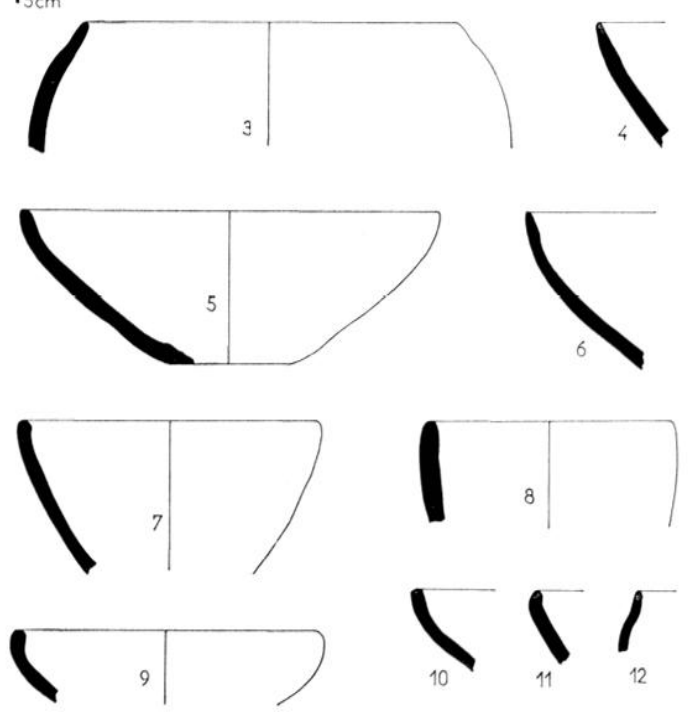

28 liebords de bols de la fosse 2.
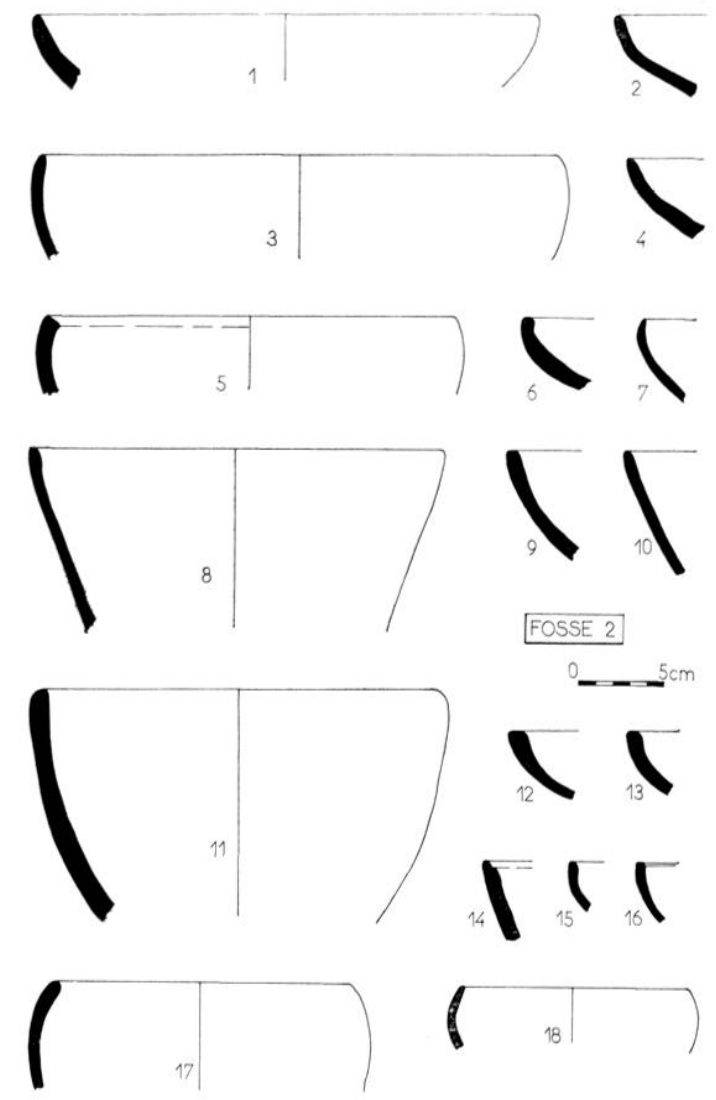

27 Rebords de bols de la fosse 2.
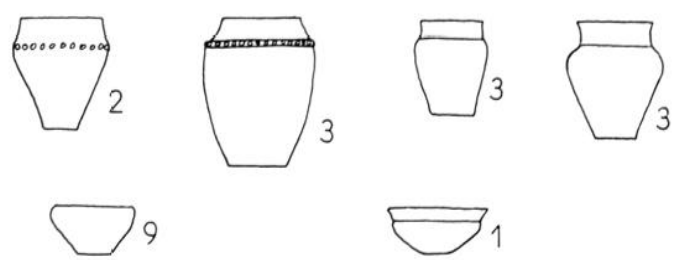

CERAMIDUE DE LA FOSSE 1
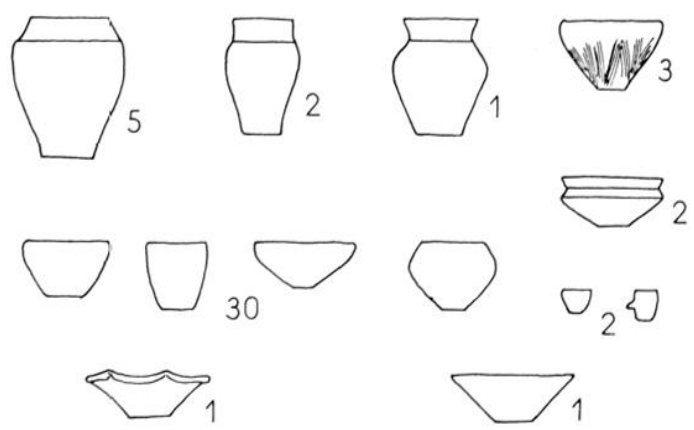

CERAMIDUE DE LA FOSSE 2

29 Tableau récapitulatif des différentes formes céramiques reconstituces des fosses 1 et 2 . 
Essayons d'interpréter le rôle de ces fosses, qui de prime abord ne semblent pas être de simples silos, foyers ou "fonds de cabane». Nous tenterons ensuite d'établir des corrélations entre ces deux fosses.

La fosse $n^{0}$ 1. Elle est caractérisée par sa forme particulière et dans la plus grande partie de ses remplissages, par les témoignages d'une forte cuisson et mème d'une calcination intense : scories, parois cuites, sols calcinés, galets éclatés. Yous sommes donc en présence d'un four. Les nombreux tessons déformés, vrilles, raquelés ou rendus poreux par une trop forte cuisson nous font adopter l'hypothèse d'un four de potier.

Nous avons précédemment mis en relief les trois types de structures aménagées successivement dans cette fosse de forme surprenante, puisqu'a priori aucun système d'aération par la base n'avait été conçu. La forme générale en trone de cône pouvait avoir pour but, d'une part la réflection de la chaleur, d'autre part la création d'un courant de convexion activant le tirage. Cette hypothèse reste à vérifier expérimentalement. Les foyers successifs, placés au centre de la fosse, ont été recouverts de galets ou de plaquettes calcaires et gréseuses, ceci peut-être en vue de régulariser la source de chaleur.

Dans un premier stade, le four semble avoir fonctionné tel que; le foyer était placé sur le fond plat de la fosse. Le système ne semble pas avoir été satisfaisant; on a alors surajouté, au centre de la fosse, un amas d'argile (IX a). Sur cette deuxième structure repose la couche VIII, de morphologie encore différente. I)ans ce dernier stade le four paraît avoir été nettoyé régulièrement. Seuls subsistent les sols calcinés (VII, VI, etc.). Ces sols correspondraient alors à un fonctionnement optimum du four.

Dans les trois phases consécutives d'aménagement, le foyer était situé au centre de la fosse : sur un fond plat, puis sur une lentille convexe, enfin dans une dépression. Les différents aménagements portent donc sur la recherche d'un rapport optimum cuisson-tirage entre le foyer central et une banquette périphérique destinée, semble-t-il, à recevoir les vases à cuire. De toute façon, nous pourons éliminer avec certitude la possibilité d'une superstructure avec sole perforée et coupole.

La fosse $n^{\circ} 2$. Elle est de morphologie toute différente. Sa forme est celle d'un ovale allongé ; ses bords évasés présentent des gradins ; la partie profonde excentrée est située à l'est. Les niveaux VII et VII b témoignent, ici aussi, d'un feu soutenu et l'utilisation en four (tessons vitrifies ou calcinés) est probable. Dans un premier temps les trous de poteau, contemporains de la première utilisation de la fosse, délimitent un enclos quadrangulaire et peuvent avoir un rôle précis pour l'utilisation du four. Nous sommes donc en présence d'un type de four différent du F1. Il n'cst pas possible de faire intervenir l'existence d'une sole perforée, mais une superstructure en matière périssable (trous de poteau) peut avoir été conçue pour favoriser le tirage.

L'emploi de ce four semble avoir été d'une durée plus courte que celui de F1; peut-être a-t-il été plus soigneusement nettoyé ? Après une période d'abandon total (VI c), une seconde utilisation est marquée par les niveaux VI et V. Nous avons déjà montré que ces niveaux lenticulaires à galets éclatés, à fort pendage, correspondaient à un dépotoir. Les éléments constitutifs de ces lentilles sont des galets éclatés par le feu, des tessons calcinés ou vrillés et des fragments d'argile cuite : ces déblais proviennent vraisemblablement 
du nettoyage d'un four voisin. Ceci est prouvé par des tessons de F1 qui se raccordent avec des tessons de F2, dans les couches VI et V.

Le tableau ci-dessous met en évidence les rapports entre les fosses 1 et 2 et les sols d'habitat extérieurs aux fosses. Ce schéma a été établi d'après les observations sur la stratigraphie, les structures et les raccords qui existent entre une même céramique brisée et dispersée dans les deux fosses. Le tableau corrélatif permet de définir six phases dans le gisement archéologique.

Ghronologie des structures

\begin{tabular}{|c|c|c|c|c|c|}
\hline STADES & $\begin{array}{l}\text { STRATI- } \\
\text { GRAPHIE }\end{array}$ & Fosse 1 & $\begin{array}{l}\text { STrati- } \\
\text { GRAPHIE }\end{array}$ & Fosse 2 & $\begin{array}{c}\text { Chrono- } \\
\text { LOGIE }\end{array}$ \\
\hline 1 & & Sol 1. & $\begin{array}{l}\text { VII } \\
\text { VII. b }\end{array}$ & $\begin{array}{c}\text { Creusement } \\
\text { fosse et poteaux, } \\
\text { utilisation en four. }\end{array}$ & 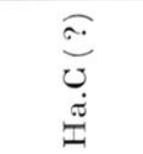 \\
\hline 2 & & & VI. c & $\begin{array}{c}\text { Abandon } \\
\text { niveau stérile. }\end{array}$ & \\
\hline 3 & $\begin{array}{r}\text { IX } \\
\text { VIII }\end{array}$ & $\begin{array}{c}\text { Creusement fosse, } \\
\text { utilisation } \\
\text { en four non curé. }\end{array}$ & & Sol 2. & $\vec{a}$ \\
\hline 4 & $\begin{array}{r}\text { VII } \\
\text { II }\end{array}$ & $\begin{array}{l}\text { Utilisation } \\
\text { en four curé. }\end{array}$ & $\begin{array}{r}\text { VI } \\
\text { V }\end{array}$ & $\begin{array}{l}\text { Utilisation } \\
\text { en dépotoir }\end{array}$ & 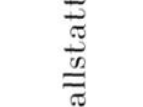 \\
\hline 5 & $\begin{array}{r}\text { III } \\
\text { II }\end{array}$ & $\begin{array}{c}\text { Niveau } \\
\text { stérile et habitat. }\end{array}$ & $\begin{array}{l}\text { IV } \\
\text { II }\end{array}$ & $\begin{array}{l}\text { Niveau } \\
\text { stérile ct habitat. }\end{array}$ & - \\
\hline 6 & I & Abandon habitat. & I & Abandon habitat. & \\
\hline
\end{tabular}

Chronologie comparée des structures des fosses 1 et 2 .

1 : creusement de la fosse 2 et utilisation en four. Formation du sol extérieur inférieur ;

2 : abandon de l'habitat. I)épòt de colluvions stériles sur toute la surface du gisement;

3 : creusement de la fosse 1 et première utilisation de ce four, sans nettoyage. Formation du sol extérieur supérieur;

4: nettoyage du four 1 entre chaque utilisation. Les déblais servent à remblayer la fosse 2;

5 : abandon du four 1. Les deux fosses ne sont plus utilisées que comme foyers ou dépotoirs d'un habitat tout proche;

6 : abandon de l'habitat et comblement total des fosses par des colluvions stériles.

Il est très probable que les dépressions qui perforent les sols extérieurs aux fosses soient des petites cuvettes d'extraction d'argile, soit pour le façonnement de poteries, soit pour la confection des banquettes d'argile autour des foyers dans les fours.

Nous disposons donc de deux fours quasi contemporains, qui appartiennent à deux types différents : la fosse 1 est une fosse circulaire à fond plat et parois surplombantes ; 
la fosse 2 est une curelte à gradins successifs et une paroi surplombante. Dans les deux cas, il n'y a ni sole perforée, ni voùte ou superstructure, ni système d'aération par la base. La chaleur est fournie par un foyer, dont l'ardeur est tempérée par des plaquettes de calcaire ou de grès ou par des galets siliceux. Les vases sont placés en bordure du foyer, sur une banquette d'argile. Des éléments de terre cuite pourraient être des supports de cuisson.

Yous avons cherché des comparaisons à ces fours dans les gisements protohistoriques de la France du nord-est. Seul le four de Marlenheim-Fessenheim (Bas-Rhin), fouillé par R. Forrer, possédait une sole d'argile cuite perforée et une coupole avec cheminée d'aération. Ce type de four à chambre de cuisson semble réservé à une vaisselle de qualité, telle qu'en a livré le four de Marlenheim. Le plus souvent, des fosses équivalentes de celles de Florange ont été interprétées en "fonds de cabane", foyers en fosse ou silos réutilisés en foyers. Jans plusieurs cas, pourtant, l'utilisation de ces fosses en fours de potier a été bien mise en évidence, mais les auteurs admettaient souvent l'existence de superstructures (sole, coupole) en argile, détruites par les labours profonds. A Florange, nous pouvons affirmer qu'il n'existait pas de superstructure.

Des fours de type F.1, circulaires à parois surplombantes, ont été fouillés à Achenheim (Bronze final), Ecury-le-Repos ("les Terres Grasses", groupe I3; Ifallstatt moyen), Strasbourg-(Conenbourg 'Bronze final), Neewiller (Ilallstatt) et Taponas (Rhòne; non daté, protohistorique?). A Necwiller, des torches en argile (anneaux de suspension) et à Strasbourg-Cironenbourg, des prismes triangulaires en argile, pouvaient assurer un support aux vases ou séparer les assiettes empilées pendant la cuisson. A Ecury-le-Repos ("les Terres (irasses", groupe A ; Hallstatl moyen) une fosse à cuvettes emboittées et foyer latéral pourrait être un four de type F.2.

De toute manière, l'utilisation des fours à coupole et soles perforés paraît tardive et ne s'est developpée qu'à Ia T'ène. Il est possible que de nombreuses fosses protohistoriques interprétés comme fonds de cabane arec traces de torchis et d'argile cuite soient bien, le plus souvent, des fours comparables à ceux de Florange.

\section{LE MOIBLIER ARCHÉOLOGIQUE : COMPARAISONS ET DATATION.}

Ce matériel n'est pas très différent d'une fosse à l'autre, ni même d'un niveau à l'autre. Il nous a paru utile de décrire plus précisément tous les objets, car la chronologie des différentes phases d'utilisation des fosses parait courte; nous pouvons donc estimer que ces objets, à quelques dizaines d'années près, sont contemporains.

\section{A. Induslrie lithique.}

Le débitage du silex n'est représenté que par huit pièces. La matière première est d'origine diverse : chaille de hase du bajocien; silex en plaquettes roulées ; petits nuclei sphériques (galets?).

L'existence d'un dos réservé sur plusieurs éclats montre bien la faible dimension des rognons de silex utilisés (figr. 10 , nos 1 et $う$; figr. 21 , nos 8 à 10 ). 
Les éclats, quelquefois laminaires, sont épais; les bulbes de percussion souvent esquillés sont très proéminents ; ces éclats ont été probablement détachés avec un percuteur de pierre. La retouche est écailleuse, souvent abrupte et se limite généralement à la bordure de l'outil : grattoir ou racloir (fig. 10, no 1 ; fig. 21 , nos 8 et 10 ).

Un couteau à dos réservé possède quelques écailles d'utilisation sur son tranchant (fig. $10, n^{0}$ )). La pièce no 3 (fig. 10) est une chute de nucléus à lamelle.

L'industrie lithique est très pauvre : il ne s'agit que d'un aménagement sommaire d'éclats débités au percuteur de pierre. Il n'est pas possible de concevoir que seul le silex soit utilisé pour la fabrication d'outils, vraisemblablement ce n'est qu'un outillage complémentaire.

Meules. Deux fragments de meules dormantes proviennent du dépotoir de la fosse 2. Elles ont été aménagées par bouchardage sur des blocs de grès roulés. On considère, en général, que l'existence de meules à grain témoigne d'activités agricoles.

\section{B. Objets métalliques.}

Le fer n'est représenté que par un résidu de coulée (fig. 22, no 4 ) et une herminette à légers ailerons terminaux (fig. 22, $n^{\circ} 1$ ). Les herminettes à ailerons apparaissent dès le Hallstatt ancien (Hallstatt (i de Reinecke), dans la nécropole de Hallstatt, associées aux grandes épées de bronze ou de fer; leur utilisation est connue jusqu'au Hallstatt final (Hallstatt I)2 de Reinecke), dans le site du Mont-Lassois.

Une aiguille en bronze (fig. $22, n^{0} 3$ ) est brisée au niveau du chas. Le chas est formé de deux perforations coniques opposées par leur sommet. L'utilisation courante de ce type d'aiguille, connu dès la fin de l'Âge du Bronze, ne se développe qu'au Hallstatt moyen.

Un bracelet à jonc torique (fig. $22, n^{0} 2$ ) est d'un type assez rare : les extrémités sont arrondies, le jonc est décoré de groupes d'incisions transversales peu profondes. En contexte Hallstatt final, nous connaissons un bracelet semblable au Mont-Lassois.

\section{Céramique.}

Sur l'ensemble des tessons, nous distinguerons immédiatement deux types de cuisson : tessons poreux, légers, avec vacuoles intérieures. Les surfaces sont souvent rouges ou orangées, quelquefois grises. I)es fragments vrillés sont dus à l'allaissement pendant la cuisson. Ils proviennent des niveaux d'utilisation des fours ou du dépotoir de la fosse 2 . Ce sont des vases brisés ou affaissés en cours de cuisson ou bien des tessons restés longtemps en bordure des foyers; - tessons plus lourds, souvent feuilletés, en terre grasse et peu cuite. Ce sont des fragments de vases cuits normalement dans les fours et brisés après utilisation. Ils proviennent des niveaux supérieurs de réemploi des fours ou du dépotoir de la fosse 2 .

Les dégraissants utilisés sont très divers. Aussi n'est-il guère possible de distinguer une céramique fine à petit dégraissant d'une céramique grossière à gros dégraissant. Les éléments les plus fréquents sont les sables micacés, calcaires ou siliceux, les quartz pilés et plus rarement les coquilles concassées.

Aucun vase n'est tourné. Ians deux cas au moins, il est assuré que des bols ont été montés au colombin. L'épaisseur moyenne des parois varie de 5 à $12 \mathrm{~mm}$ et seules les 
parois de jattes ou d'assiettes n'excèdent pas 5 mm. Les surfaces extérieures peuvent être de couleur grise, beige, mais souvent brunâtre, rouge ou noire. Dans la plupart des cas, elles sont simplement lissées au doigt. La technique du lustrage est très rare. Une grosse urne carénée (fig. 11), possède un col soigneusement lissé ; la partie inféricure, en dessous de la carène, est laissée volontairement brute (opposition ombre-lumière). Ces techniques ne varient guère en fonction des types de céramique et il n'existe presque pas de différence entre les petits récipients (jattes, bols, ete.) et les grosses céramiques utilitaires (urnes, jarres).

Les formes céramiques sont assez variées mais seuls les bols et les urnes existent en grand nombre. Les bols (38 vases) sont à fond plat et à bord droit ou rentrant (fig. 14,26 et 28 ); - les urnes (19) vases au total) appartiennent à 4 types bien différenciés : $1^{\circ}$ urne carénée à bord rentrant $\{8$ vases $)$. Ln cordon ou des impressions digitales soulignent la carène (fig. 11). II. E. Mariën signale une urne de ce type dans la nécropole de "De Hamert ", vers Venlo (Pays-Bas), en contexte Hallstatt C -- I)1; 20 urne carénée à bord droit (5 vases) (fig. 13, nos 10 et 11). I)ans le cimetière hallstattien (HA.D1) de Court-SaintÉtienne (Belgique), l'urne no 43 en forme de situle apparaît à la fin du Hallstatt C; $3^{\circ}$ urne voisine du type de "Laufeld" (4 vases) (fig. 13, nos 1 et 2). Les exemplaires de Laufeld, dans la région de Dusseldorf, sont plus trapus. Mais à partir du Hallstatt D, les formes deviennent élancées ainsi que le remarque II. E. Mariën à propos d'un vase ( $\left.n^{0} 40\right)$ de Court-Saint-Étienne; $4^{\circ}$ urne voisine du type de "Harpstedt", à bord lisse et non crénelé (2 vases) (fig. 12). Le type de "Harpstedt " est représenté jusqu'au Hallstatt I).1.

Des jattes carénées à bord déversé ou à profil en $S$ (3 vases) (fig. 14, nos 7 et 26 , nos 9 et 10) apparaissent dès la fin de l'Âge du Bronze. Elles sont encore représentées à La Tène ancienne. Deux micro-rases, en forme de bol (fig. 21 , nos 2 et 3 ), sont de fabrication soignée. L'un est entièrement lustré. Du mème niveau, deux vases-biberon en terre cuite (fig. 21, nos 1 et 4). Ces récipients sont connus aussi bien à l'Âge du Bronze final qu'au Hallstatt. Lne coupe conique possède un rebord simple légèrement biseauté (fig. 26, no 2). Dans le dépotoir de la fosse 2 , nous avons recueilli quatre tessons d'une coupe à indentations (fig. 24). Quelques coupes existent dans les briquetages de Bretagne. Les exemplaires les plus proches, du Hallstatt (i ou I), se trouvent en Belgique (Vosselar, Anvers).

Parmi les petits objets en terre cuite, nous avons dénombré un peson (fig. 13, no 12) et trois fusaïoles ou grains d'enfilage (fig. $21, n^{0 \text { o }}$ j à 7 ).

Les décors ne se trouvent que sur des bols ou des urnes : décors incisé en chevrons (fig. 23, en bas à gauche); décor peigné verticalement (fig. $23, \mathrm{n}^{\text {os }} 1$ à 7 ); décor peigné en oves (fig. $23, \mathrm{n}^{\circ}$ ) ); impressions digitales en lignes horizontales sur le fond, sur la carène ou sous le rebord des urnes (type de "Harpstedt"); décor plastique par pastille (fig. 10, $n^{0} 8$ ) ou par cordons horizontaux à impressions digitales nettement séparées.

En pourcentage, seul $1 / 4$ de la céramique porte des décors. Nous n'avons trouvé aucune trace de décor peint ou graphité, largement répandu en Alsace et en Bourgogne.

La recherche d'éléments de comparaison à la céramique de Florange nous conduit à éliminer des influences du Hallstatt ancien (faciès de Koberstadt ou Bourguignon) ou du Hallstatt final - La Tène ancienne ( Vix, Jogasses, Hunsück-Eifel). Les éléments 
les plus proches se trouvent en Belgique, en IIollande ou dans la basse vallée du Rhin, en contexte Iallstatt moyen (IIa. C-D)1 de Reinecke).

Un échantillon de charbon de bois, prélevé dans le niveau inférieur (IX) de la fosse $\mathrm{n}^{0}$ 1, a été daté au Laboratoire des Faibles Radioactivités de Gif-sur-Yvette (G. I)elibrias). Le résultat de la mesure d'âge est le suivant (échantillon no GIF-1813) :

$200 \pm 70$ ans, soit 50 environ avant .J.-(

Cette datation correspond parfaitement à ce que l'on sait du mobilier archéologrique recueilli dans les deux fours de Florange.

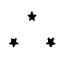

Au niveau de Thionville, les influences culturelles septentrionales paraissent prépondérantes au Premier İge du Fer. Le faciès hallstattien mis en evidence à Florange n'a que peu de rapports avee les faciès du Hallstatt ancien ou moyen d'Alsace, de FrancheComté ou de Bourgogne, romme avec le faciès Hallstatt final du Mont-Lassois ou des Jogasses. En Lorraine, J.-P. Millotte a mis en évirlence l'existence d'un Mallstattien ancien original à Récicourt et Yeuvilly (Meuse), qui, par la vallée de la Moselle, pent avoir comene origine les Pays-Bas et la balse vallée du Rhin. La foulle de Florange nous montre probablement un aspect plus tarlif de cette civilisation au llallstatt moyen. Une fouille précise dans des remplissages aisés a travialler a permis deuturlier les structures de deux fours de potier. Cies dernières sont interessantes en elles-mêmes, car rares sont les fours protohistoriques bien ronnus. Ia découverte de ces fours et dépotoirs permet d'envisager l'existence d'un habitat contemporain tout proche, qui reste à trouver et à explorer.

P. Pítrequin, J.-F. Pringre, D. Vualdat.

BHBLiOgRAPHI:

Brissox (A.) et IIate (J.-J.), Fonds de cabane de l'ìge du Bronze final et du premier îge du Fer en Champagne, dans Recue arch. de l'Esl, XVIII, 1967, p. 7-51.

Comminer (J.), Le fond de cabane de Taponas (Rhòne), dans Revue arch. de l'Est, I, 1950, p. 21:3247.

Favnet (P.--II.), Les nécropoles des Jogasses it Chouilly, dans Préhistnire, V, Paris, 1936, p. 24119.

Jorm:k (R.), Jän Hallstall Jöpfirofen hei Vartenhein Fessenheim. dants Anzeiger für Elsässische Allertamstiunde, 1915, p. 504-510.

IIATt (J.-J.), Découverte à Achenheim d'un four à potier de la période des Champs d'urnes, danls Cahiers d'archéo. et d'histoire d'Alsace, no 132, 1952, p. 43-53.3.

Hatr (J.-J.), Chronique de Prolohistoire, VI. Pour une nouvelle chronologie de l'époque hallstattienne, les trois phases du Ier àge de fer en Allemagne du sud el en lirance de l'est, dans Bulletin Soc. J'réhist. Fr., LIX, 1962, p. 6599-6667.

Hatr (J.-J.) et Zcwstrix (II.j, Découverte d'un four de polier de l'ige du Bronze final a Cronenbourg (Bas--Khin), dans Cahiers alsaciens d'arch.. d'art et d'histoire, IV, 1960, p. 17-266. 
Jofrroy (R.), L'oppidum de Vix et la civilisation hallstattienne finale dans l'est de la France, Paris, 1960.

Mariëx (II. E.), Oud-België, Anvers, 1952.

MariËx (II. E.), Trouvailles du Champ d'urnes et des lombelles hallstaltiennes de Court-Saint-Étienne, Monographies d'Archéologie Royale, I, Bruxelles, 1958.

Mrrlotre (J.-P.), Le Jura et les plaines de Saône aux Ầges des métaux, Annales Littéraires, Université de Besançon, 59 (Archéologie, 16), 2 vol., Paris, 1963.

Millotte (J.-P.), Carte archéologique de la Lorraine (Âges du Bronze el du Fer), Annales Littéraires, Université de Besangon, 7.3 (Archéologie, 18), Paris, 1865.

Millotre (J.-P.), Précis de prolohistoire européenne, Coll. U2, Paris, 1970.

PARET (O.), Lrgeschichte Würtlembergs, Stuttgart, 1921, p. 83.

Schasfrer (C. F. A.), Un four de potier hallstattien à Neewiller (Bas-Rhin), dans Cahiers d'arch. el d'hist. d'Alsace, nos $53-56,1923$, p. 77-84.

stramfuss (R.), Siedlungsfunde der jüngeren Bronze und älteren kisenzeit im westlichen Ruhrgebiet, dans Quellenschriften zur westdeutschen Vor und Frühgeschichte, 7, Bonn, 1959.

Tackixibrg (K.), Die Kultur der frühen Eisenzeit in Miltel - und Westhannover, Leiprigr, 1934. 